\title{
THE TOPOLOGY OF ISOPARAMETRIC SUBMANIFOLDS
}

\author{
WU-YI HSIANG, RICHARD S. PALAIS \& CHUU-LIAN TERNG
}

\begin{abstract}
It has been known since a famous paper of Bott and Samelson that, using Morse theory, the homology and cohomology of certain homogeneous spaces can be computed algorithmically from Dynkin diagram and multiplicity data. L. Conlon and J. Dadok noted that these spaces are the orbits of the isotropy representations of symmetric spaces. Recently the theory of isoparametric hypersurfaces has been generalized to a theory of isoparametric submanifolds of arbitrary codimension in Euclidean space, and these same orbits turn out to be exactly the homogeneous examples. Even the nonhomogeneous examples have associated to them Weyl groups with Dynkin diagrams marked with multiplicities. We extend and simplify the Bott-Samelson method to compute the homology and cohomology of isoparametric submanifolds from their marked Dynkin diagrams.
\end{abstract}

\section{Introduction}

In 1958 Bott and Samelson introduced the concept of variational completeness for isometric group actions [5], and developed powerful Morse theoretic arguments to compute the homology and cohomology of orbits of variationally complete actions. As already noted in their paper, the isotropy representations of symmetric spaces ( $s$-representations) are variationally complete, and from results of L. Conlon [19], [20] an orthogonal representation is variationally complete if and only if there is a linear subspace which meets every orbit orthogonally. Such representations (called polar by J. Dadok [21] and representations admitting sections by Palais and Terng [40]) have been classified by Dadok, who showed that, at least as far as orbit structure is concerned, they are exactly the $s$-representations. This class of homogeneous spaces includes all the flag manifolds and Grassmannians, and because of its important roles in geometry, topology, and representation theory it has been intensively studied. We shall see below that isoparametric submanifolds and their focal manifolds are a geometric generalization of these homogeneous spaces.

Received January 21, 1986 and, in revised form, April 22, 1987. Research was supported in part by National Science Foundation grants MC 577-23579 (Hsiang), MCS8102696 (Palais) and DMS-8301928 (Terng). The second author was also supported by the Mathematical Sciences Research Institute. 
A smooth function $f$ on a space form $N^{n+1}(c)$ is called isoparametric if $\Delta f$ and $|\nabla f|^{2}$ are functions of $f$. It is easily seen that, for a regular value $a, f^{-1}(a)$ is a hypersurface of $N^{n+1}(c)$ having constant principal curvatures; such hypersurfaces are called isoparametric. The problem of determining all isoparametric functions is called the "Levi-Civita Problem" [16] and was first studied by Levi-Civita [33] for $\mathbb{R}^{3}, B$. Segré [42] for $\mathbb{R}^{n+1}$, and by É. Cartan [7] for $H^{n+1}$ and $S^{n+1}$. In a series of papers [7]-[10] in the late 1930's Cartan was fascinated by the complexity of the spherical case and its mysterious connections with Lie theory. This subject was then somehow forgotten until it was revived by a paper of Nomizu [36] and two remarkable papers of Münzner [35]. It is easily seen that a principal orbit of an orthogonal representation with two-dimensional orbit space is isoparametric in the unit sphere. Such orthogonal representations were classified by Hsiang and Lawson [28]; they are exactly the isotropy representations of the symmetric spaces of rank two. However there are also infinitely many families of isoparametric hypersurfaces in spheres constructed by Ozeki and Takeuchi [37] and Ferus, Karcher and Münzner [23], which are not orbits.

The relation between isoparametric hypersurfaces and symmetric spaces now seems apparent enough that in retrospect it appears surprising that the master of both fields missed realizing that it pointed to a more general isoparametric theory for arbitrary codimensions. Suppose $M^{n}$ is a principal orbit of an orthogonal representation $\rho: G \rightarrow \mathrm{O}(n+k)$, and $v_{0}$ is a normal vector to $M^{n}$ in $\nu(M)_{x_{0}}$. Then $\tilde{v}_{0}\left(g x_{0}\right)=d g_{x_{0}}\left(v_{0}\right)$, is a well-defined, $G$-invariant, global normal vector field on $M$. It is obvious that the shape operator $A_{\tilde{v}_{0}\left(g x_{0}\right)}$ is orthogonally conjugate to $A_{v_{0}}$, so the principal curvatures of $M^{n}$ in the directions $\tilde{v}_{0}(x)$ are constant (i.e., independent of $x$ ). But in general the $G$ equivariant normal field $\tilde{v}_{0}$ is not parallel in the Riemannian sense when $M^{n}$ is regarded as a submanifold of $\mathbb{R}^{n+k}$. Indeed the condition that $G$-equivariant normal fields are Riemannian parallel is equivalent to the condition that $\rho$ is polar [40]. Now let $G / K$ be a symmetric space of rank $k, \mathfrak{g}=\mathfrak{K}+\mathfrak{P}$ the Cartan decomposition, and $M=K x$ a principal orbit of the isotropy representation of $K$ on $\mathfrak{P}$. Then $\tau=\nu(M)_{x}$ is a maximal abelian subalgebra (Cartan subalgebra) in $\mathfrak{P}$, and $M$ is a codimension $k$ submanifold of $\mathfrak{P}$ which satisfies the following conditions: (i) its normal bundle is flat (because $K$-equivariant normal fields are parallel), and (ii) the principal curvatures with respect to any parallel normal field are constant. Now for any parallel normal field $v$ the parallel set, $M_{v}=\{x+v(x) \mid x \in M\}$, is a $K$-orbit. So the parallel foliation, $\left\{M_{v}\right\}$, is just the orbit foliation of $K$ acting on $\mathfrak{P}$. Moreover the union of the singular orbits is exactly the focal set of $M^{n}$ in the sense of Morse theory. There is a natural Weyl group $W$ acting on $\tau$ so that $\mathfrak{P} / K \approx \tau / W$, and 
the multiplicity $m_{i}$ is defined for each reflection hyperplane $l_{i}$ of $W$ to be the multiplicity of the focal points $x \in l_{i} \backslash\left\{\bigcup l_{j} \mid i \neq j\right\}$. In fact these multiplicities are also the differences in dimension of a principal orbit and subprincipal orbits of $\mathfrak{P}$. Motivated by these examples Terng in a recent paper [44] defined a submanifold $M^{n} \subset \mathbb{R}^{n+k}$ to be isoparametric if it satisfied (i) and (ii) above. Then it was shown in [44] that these general isoparametric submanifolds and their focal manifolds share many of the geometric properties of orbits of isotropy representations of symmetric spaces. In particular there is again a Coxeter group and associated multiplicities (in general different from the homogeneous examples) that generalizes the fundamental structure of the Weyl group action on a chosen Cartan sublagebra. Indeed, short of homogeneity itself, almost every general geometric or topological property of the principal orbits of isotropy representations of symmetric spaces seem to hold also for these isoparametric submanifolds.

In this paper we prove in particular that the theorems of Bott and Samelson (and even many of their Morse theoretic techniques), computing the homology and cohomology structure of these orbits, carry over to the general isoparametric case. Lacking $G$ we must replace certain group theoretic methods of Bott and Samelson (such as the construction of their iterated sphere bundles $\Gamma_{s}$ ) by Riemannian geometric substitutes, but surprisingly this seems to simplify and clarify the argument. As another consequence we are able to generalize Borel's theorem on the rational cohomology of $G / T$ to isoparametric submanifolds with even multiplicities.

This paper is organized as follows: in $\S 1$ we recall the definitions and basic properties of isoparametric submanifolds and in $\S 2$ we prove an analogue of the slice representation theorem for polar representations for general isoparametric families, and using this slice theorem and a result of Münzner we prove that Coxeter groups associated to an isoparametric submanifold are crystallographic and also give restrictions on their possible marked Dynkin diagrams. In $\S 3$ we obtain explicitly the marked Dynkin diagrams for all homogeneous isoparametric submanfolds. In $\S 4$ we develop the basic Morse theory we need for the computation of the homology and cohomology of isoparametric submanifolds, and in $\S \S 5$ and 6 we carry out this computation.

\section{Known properties of isoparametric submanifolds}

A compact submanifold $M^{n}$ of $\mathbb{R}^{n+k}$ is called isoparametric if its normal bundle $\nu(M)$ is flat and has trivial holonomy (the latter is in fact automatic), and if given a parallel section $v$ of $\nu(M)$, the principal curvatures of $M$ at $x$ in the direction $v(x)$ are independent of $x$. Below we develop some notations 
and state certain properties of $M$ which are proved in [44]. Without loss of generality, we can assume $M$ is full, i.e., not included in any proper affine subspace of $\mathbb{R}^{n+k}$.

1.1. $M$ is included in a standard sphere of $\mathbb{R}^{n+k}$. Hence we may assume that $M$ is contained in the unit sphere centered at the origin in $\mathbb{R}^{n+k}$, so that all the affine normal planes $\left(x+\nu(M)_{x}\right), x \in M$, are linear subspaces of $\mathbb{R}^{n+k}$. Since the holonomy of $\nu(M)$ is trivial, for each $x, x^{\prime}$ in $M$ we have a canonical parallel translation map $\Pi_{x, x^{\prime}}: \nu(M)_{x} \rightarrow \nu(M)_{x^{\prime}}$.

1.2. $T M$ has a canonical splitting as the orthogonal direct sum of $p$ subbundles $E_{i}$, each of which is integrable, say of fiber dimension $m_{i}$, and has associated to it a canonical parallel normal field $v_{i}$ (the $i$ th curvature normal vector). These are related and in fact characterized by the following fact: the set $\left\{A_{v}\right\}$ of shape operators, $v \in \nu(M)_{x}$, is a commuting family of self-adjoint operators on $T M_{x}$ and the $E_{i}(x)$ are the common eigenspaces with corresponding eigenvalues $\left\langle v, v_{i}(x)\right\rangle$, i.e., the shape operator $A_{v}$ is given by $\sum\left\langle v, v_{i}\right\rangle \operatorname{id}_{E_{i}}$. These $E_{i}$ 's are called the curvature distributions of $M$ and the $m_{i}$ 's are called the multiplicities of $M$.

1.3. The leaf $S_{i}(x)$ of the integrable subbundle $E_{i}$ through $x \in M$ is a standard $m_{i}$ dimensional sphere in $\mathbb{R}^{n+k}$ (centered at $\left.x+\left(v_{i}(x) /\left\langle v_{i}, v_{i}\right\rangle\right)\right)$.

1.4. In each affine normal plane $x+\nu(M)_{x}$ we have $p$ linear hyperplanes $l_{1}(x), \cdots, l_{p}(x)$; namely $l_{i}(x)$ is the linear hyperplane of $x+\nu(M)_{x}$ orthogonal to the curvature normal $v_{i}(x)$. In fact $\left(x+v_{0}\right) \in l_{i}(x)$ if and only if $\left\langle v_{0}, v_{i}(x)\right\rangle=1$. Since the parallel translation $\Pi_{x, x^{\prime}}$ is orthogonal and maps $v_{i}(x)$ to $v_{i}\left(x^{\prime}\right)$, it maps $l_{i}(x)$ to $l_{i}\left(x^{\prime}\right)$. Let $Y: \nu(M) \rightarrow \mathbb{R}^{n+k}$ be the normal bundle map (i.e., $Y(v)=x+v$ for $\left.v \in \nu(M)_{x}\right)$ and $C(Y)$ the set of all singular points of $Y$. Then the set $\Gamma$ of focal points of $M$ is by definition $Y(C(Y))$. $\Gamma$ intersects $\left(x+\nu(M)_{x}\right)$ in the union of the hyperplanes $l_{1}(x), \cdots, l_{p}(x)$, and $C(Y)=Y^{-1}(\Gamma)$.

1.5. The reflections $R_{i}^{x}$ of $x+\nu(M)_{x}$ in the hyperplanes $l_{i}(x)$ permute these hyperplanes; $R_{i}^{x}\left(l_{j}(x)\right)=l_{\rho^{i}(j)}(x)\left(\rho^{i} \in S_{p}\right.$, the symmetric group on $p$ elements), and $v_{1}(x), \cdots, v_{p}(x)$ spans $\nu(M)_{x}$. Thus the group $W^{x}$ generated by these reflections is a finite Coxeter group of rank $k$, and we have a faithful representation $\rho: W^{x} \rightarrow S_{p}$ with $\rho\left(R_{i}^{x}\right)=\rho^{i}$. The curvature normal vectors $v_{j}(x)$ are a root system for $W^{x}$. Moreover the parallel translation map $\Pi_{x, x^{\prime}}: \nu(M)_{x} \rightarrow \nu(M)_{x^{\prime}}$ conjugates $W^{x}$ to $W^{x^{\prime}}$, so we have associated to $M$ a well-defined Coxeter group $W$. The multiplicities $m_{i}$ are invariant under $W$ (in the sense that $m_{i}=m_{\rho^{j}(i)}$ ). It follows that to an isoparametric submanifold $M$ of codimension $k$ we can associate a well-defined marked Dynkin diagram with $k$ vertices, namely the Dynkin diagram of $W$ marked with the multiplicities $m_{i}$. 
1.6. The Coxeter group $W$ acts freely on $M$ as a group of diffeomorphisms. The reflection $R_{i}$ of $W$ corresponds to the diffeomorphism $\varphi_{i}$ of $M$, which maps $x \in M$ to $x^{\prime}=\varphi_{i}(x)$, where $x^{\prime}$ is the antipodal point to $x$ on the sphere $S_{i}(x)$ (the leaf of $E_{i}$ through $x$ ). Moreover $\varphi_{i}^{*}\left(E_{j}\right)=E_{\rho^{i}(j)}$.

1.7. Let $U$ be a connected component of the complement of the union of the hyperplanes $l_{i}(x)$ in $\left(x+\nu(M)_{x}\right)$. Then its closure $\bar{U}$ is a simplicial cone, and a fundamental domain or Weyl chamber for $W^{x}$, i.e., each $W^{x}$ orbit meets $\bar{U}$ in exactly one point. For each simplex $\sigma$ of $\bar{U}$, we define the following:

$$
\begin{aligned}
& I(x, \sigma)=\left\{j \mid \sigma \subset l_{j}(x)\right\}, \\
& V(\sigma)=\bigcap\left\{l_{j}(x) \mid j \in I(x, \sigma)\right\}, \\
& \xi(\sigma)=\text { the orthogonal complement of } V(\sigma) \text { in }\left(x+\nu(M)_{x}\right), \\
& \eta(\sigma)=\bigoplus\left\{E_{j}(x) \mid j \in I(x, \sigma)\right\} \oplus \xi(\sigma), \\
& m_{\sigma}=\sum\left\{m_{j} \mid j \in I(x, \sigma)\right\}, \\
& W_{\sigma}^{x}=\text { the subgroup of } W^{x} \text { generated by the } R_{j} \text { with } j \in I(x, \sigma), \\
& \Delta_{x}=\text { the Weyl chamber containing } x .
\end{aligned}
$$

1.8. Let $q \in\left(x+\nu(M)_{x}\right)$. Then $q$ is $W$-regular if and only if $q$ is nonfocal (i.e., $q$ is not in any $\left.l_{i}(x)\right)$. Moreover if $q \in \sigma$ then its isotropy subgroup $W_{q}^{x}$ is equal to $W_{\sigma}^{x}$.

1.9. Let $v$ be a parallel normal field such that $v\left(x_{0}\right)=v_{0}$ and $q=x_{0}+v_{0}$ is a point on a simplex $\sigma$ of a Weyl chamber. Then $M_{v}=\{x+v(x) \mid x \in M\}$ is a smooth $\left(n-m_{\sigma}\right)$ dimensional submanifold of $\mathbb{R}^{n+k}$ called the parallel manifold of $M$ through $q$, and it is also denoted by $M_{q}$. In particular if $q$ belongs to a $(k-1)$-simplex $\sigma \subset l_{i}$ then $m_{\sigma}=m_{i}$, and $\operatorname{dim}\left(M_{q}\right)=n-m_{i}$. In general $M_{q}$ intersects $\mathbb{R}^{k}=\left(x_{0}+\nu(M)_{x_{0}}\right)$ in the Weyl group orbit $W \cdot q$. The set $\left\{M_{q} \mid q \in \Delta_{x_{0}}\right\}$ is a singular foliation of $\mathbb{R}^{n+k}$ with $\mathbb{R}^{k} / W=\Delta_{x_{0}}$ as leaf space. This foliation is called the parallel foliation or isoparametric foliation defined by $M$. Moreover the map $\Pi_{v}: M \rightarrow M_{v}$ defined by $\Pi_{v}(x)=x+v(x)$ is a submersion. If $q$ is $W$-regular then $M_{v}$ is isoparametric and $\Pi_{v}$ is a diffeomorphism.

1.10. Let $u_{1}, \cdots, u_{k}$ be a set of generators of the ring of $W$-invariant polynomials on $\mathbb{R}^{k}=\left(x_{0}+\nu(M)_{x_{0}}\right)$. Then there is a unique isoparametric polynomial map (Cartan polynomial) $f: \mathbb{R}^{n+k} \rightarrow \mathbb{R}^{k}$ extending $u=$ $\left(u_{1}, \cdots, u_{k}\right)$ on $\mathbb{R}^{k}$, and the level sets of $f$ are precisely the parallel manifolds of $M$, i.e., the isoparametric foliation defined by $M$ is $\left\{f^{-1}(a) \mid a \in \mathbb{R}^{k}\right\}$. (A map $f=\left(f_{1}, \cdots, f_{k}\right)$ is called isoparametric if $\Delta f_{i}$ and $\left\langle\Delta f_{i}, \Delta f_{j}\right\rangle$ are functions of $f$ and if $\left[\nabla f_{i}, \nabla f_{j}\right]$ is a linear combination of $\nabla f_{1}, \cdots, \nabla f_{k}$ for all $i, j$.) Hence if $v$ and $v^{\prime}$ are two parallel normal fields on $M$ such that $M_{v} \cap M_{v^{\prime}}$ is not empty then $M_{v}=M_{v^{\prime}}$, i.e., there is a unique manifold $M_{q}$ parallel to $M$ through any $q$ in $\mathbb{R}^{n+k}$. 
1.11. It follows easily from the Gauss equations that the curvature tensor of $M$ can be written in terms of the $E_{i}$ 's and the $v_{i}$ 's. In fact we have $\wedge^{2}(T M)=\bigoplus\left\{E_{i} \wedge E_{j} \mid i<j\right\}$, and the Riemann tensor $R$, the Ricci tensor Ric, and the scalar curvature $R$ are given as follows:

$$
\begin{aligned}
R & =\sum_{i<j}\left\langle v_{i}, v_{j}\right\rangle \operatorname{id}_{E_{i} \wedge E_{j}}, \\
\text { Ric } & =\sum_{i}\left\langle v_{i}, H-v_{i}\right\rangle \operatorname{id}_{E_{i}}, \quad \text { where } H=\sum m_{i} v_{i} \\
R & =|H|^{2}-\sum m_{i}\left|v_{i}\right|^{2} .
\end{aligned}
$$

Since the normal bundle $\nu(M)$ is trivial and $T M=\bigoplus E_{i}$, we have the following formula for the Stiefel-Whitney classes:

$$
1=\prod\left\{\omega\left(E_{i}\right) \mid 1 \leq i \leq p\right\}
$$

1.12. $M$ is called irreducible if it cannot be written as a product of two lower dimensional isoparametric submanifolds. $M$ is irreducible if and only if the associated Coxeter group $W$ of $M$ is irreducible and we have the following decomposition theorem: every isoparametric submanifold can be written uniquely (except for order) as the product of irreducible ones.

1.13. An orthogonal representation $\rho$ of a compact Lie group $G$ is called polar if the normal plane to a principal orbit meets every orbit orthogonally. It is proved in [40] that the homogeneous isoparametric submanifolds are precisely the principal orbits of polar representations, and in fact they are principal orbits of isotropy representations of symmetric spaces (the latter always being polar). Moreover in this case the normal plane through $x$ is just the maximal abelian subalgebra through $x$ and the associated Coxeter groups and Weyl chambers are the standard ones for the symmetric space [25]. The isoparametric foliation $\left\{M_{q} \mid q \in \Delta_{x}\right\}$ is the orbit foliation, $\Delta_{x}$ is the orbit space, the simplicial decomposition of $\Delta_{x}$ is just the orbit type decomposition, and the $m_{i}$ are the differences of dimensions between principal and subprincipal orbits. The submersion $\Pi_{v}: M_{x} \rightarrow M_{y}$, with $y=x+v(x)$, is the equivariant fibration $K_{y} / K_{x} \rightarrow K x \rightarrow K y$. Hence following the convention for symmetric spaces, we will say that a full isoparametric submanifold of codimension $k$ has rank $k$.

1.14. Many nonhomogeneous isoparametric submanifolds were constructed from representations of the Clifford algebra by Ferus, Karcher and Münzner (see [23] for details). Let $\left(P_{0}, \cdots, P_{m}\right)$ be a "Clifford system" on $\mathbb{R}^{2 k}$, i.e., the $P_{i}$ 's are symmetric operators on $\mathbb{R}^{2 k}$ such that $P_{i} P_{j}+P_{j} P_{i}=2 \delta_{i j} I$. 
And let $g: \mathbb{R}^{2 k} \rightarrow \mathbb{R}$ be the degree 4 polynomial

$$
g(x)=\langle x, x\rangle 2-2 \sum_{i=1}^{m}\left\langle P_{i}(x), x\right\rangle^{2} .
$$

Then $M=g^{-1}(c) \cap S^{2 k-1}$ is isoparametric for any regular value $c$ of $g$, with $B_{2}$ (dihedral group of 8 elements) as the Coxeter group, with multiplicities $m_{1}=m$ and $m_{2}=k-m-1$, and $f(x)=(\langle x, x\rangle, g(x))$ is an isoparametric polynomial map for $M$. Most of these examples are nonhomogeneous.

\section{The Slice Theorem and determination of possible $m_{i}$ 's}

If $V$ is a polar representation of $G$, then for any $v \in V$ the "slice representation" of $G_{v}$ on the normal plane to $G v$ at $v$ is also polar [21, Theorem 3]. This allows one to study polar representations inductively. In this section we will first give an analogue for isoparametric submanifolds of the above slice representation theorem, which similarly provides an inductive method for proving many of our results. Using this inductive method and the results about codimension one isoparametric hypersurfaces in spheres (i.e., rank 2 isoparametric submanifolds) obtained by Münzner [35] and Abresch [1], we will prove that the associated Coxeter groups of isoparametric submanifolds are crystallographic (i.e., Weyl groups) and we will also give some restriction on their possible multiplicities.

2.1. Slice Theorem. We use the same notations as in 1.7 and 1.9 , put $\tilde{x}_{0}=\left(x_{0}+v\left(x_{0}\right)\right) \in \sigma$, and let $N_{x_{0}, v}$ denote the fiber of the submersion $\Pi_{v}: M \rightarrow M_{v}$ through $x_{0}$. Then

(i) $N_{x_{0}, v}$ is an $m_{\sigma}$-dimensional isoparametric submanifold of the $\left(m_{\sigma}+k\right)$ dimensional Euclidean space $\nu\left(M_{v}\right)_{\tilde{x}_{0}}$. In fact $N_{x_{0}, v} \subset\left(x_{0}+\eta\left(x_{0}, \sigma\right)\right)$ and is of $\operatorname{rank}(k-\operatorname{dim}(\sigma))$.

(ii) The normal plane to $N_{x_{0}, v}$ in $\left(x_{0}+\eta\left(x_{0}, \sigma\right)\right)$ at $x_{0}$ is $\left(x_{0}+\xi(\sigma)\right)$, the associated Coxeter group of $N_{x_{0}, v}$ is $W_{\sigma}$, and the reflection hyperplanes of $W_{\sigma}$ are $\left\{l_{j}\left(x_{0}\right) \cap\left(x_{0}+\xi(\sigma)\right) \mid j \in I\left(x_{0}, \sigma\right)\right\}$ with multiplicities $m_{j}$.

(iii) If $v^{*}$ is another parallel normal field such that $\left(x_{0}+v^{*}\left(x_{0}\right)\right) \in \sigma$, then $N_{x_{0}, v}=N_{x_{0}, v^{*}}$, so we may also denote it by $N_{x_{0}, \sigma}$.

(iv) If $y \in N_{x_{0}, \sigma}$, then $V(\sigma) \subset \nu(M)_{y}$, and the normal plane to $N_{x_{0}, \sigma}$ at $y$ in $\nu\left(M_{v}\right)_{\tilde{x}_{0}}$ is equal to $\nu(M)_{y}$.

(v) if $y \in M$ and $\left(\tilde{x}_{0}-y\right) \in \nu\left(M_{v}\right)_{\tilde{x}_{0}}$ then $\left(\tilde{x}_{0}-y\right) \in \nu(M)_{y}$.

(vi) $M \cap \nu\left(M_{v}\right)_{\tilde{x}_{0}}=\bigcup\left\{N_{x_{j}, \sigma} \mid x_{i} \in W \cdot x_{0}\right\}$, which has $|W| /\left|W_{\sigma}\right|$ connected components, and $N_{x_{j}, \sigma}$ is parallel to $N_{x_{0}, \sigma}$ in $\nu\left(M_{v}\right)_{\tilde{x}_{0}}$ through $x_{i}$. 
Proof. Let $X: M \rightarrow \mathbb{R}^{n+k}$ be the inclusion map of $M$. Then $\Pi_{v}=X+v$, and

$$
d \Pi_{v}=d X+d v=\sum\left(1-\left\langle v, v_{i}\right\rangle\right) \operatorname{id}_{E_{i}} .
$$

Since $j \in I(\sigma, x)$ if and only if $1-\left\langle v, v_{j}\right\rangle=0, \Pi_{v}$ is submersion. Then the fiber $N_{x_{0}, v}=\Pi_{v}^{-1}\left(\tilde{x}_{0}\right)$ is a submanifold of dimension $m_{\sigma}$. Now because

$$
\begin{aligned}
T\left(M_{v}\right)_{\tilde{x}_{0}} & =\bigoplus\left\{E_{j}(x) \mid j \text { is not in } I(x, \sigma)\right\}, \\
\nu\left(M_{v}\right)_{\tilde{x}_{0}} & =\bigoplus\left\{E_{j}(x) \mid j \in I(x, \sigma)\right\} \oplus \nu(M)_{x}, \\
T\left(N_{x_{0}, v}\right)_{x} & =\bigoplus\left\{E_{j}(x) \mid j \in I(x, \sigma)\right\},
\end{aligned}
$$

for all $x \in N_{x_{0}, v}$, the fixed plane $T\left(M_{v}\right)_{\tilde{x}_{0}}$ is always normal to $N_{x_{0}, v}$. Hence $N_{x_{0}, v} \subset\left(\nu\left(M_{v}\right)_{\tilde{x}_{0}}\right)$, and $\bigoplus\left\{E_{i} \mid i \in I\left(x_{0}, \sigma\right)\right\}$ is integrable with $N_{x_{0}, \sigma}$ as the leaf through $x_{0}$ and (iii) and (iv) follow. Moreover because the second fundamental form of $N_{x_{0}, v}$ in the direction $n \in V(\sigma)$ is zero and the normal bundle of $N_{x_{0}, v}$ is flat it follows that $N_{x_{0}, v} \subset\left(x_{0}+\eta\left(x_{0}, \sigma\right)\right)$. Then (i) and (ii) follow from equation $\left(^{*}\right)$ and the fact that $M$ is isoparametric.

We will prove (v) and (vi) by using 1.10. Since $N_{x_{0}, v}$ is isoparametric in $\varsigma(\sigma)=\nu\left(M_{v}\right)_{\tilde{x}_{0}}$ and $y \in \varsigma(\sigma)$, there is a parallel normal field $u$ on $N_{x_{0}, v}$ in $\varsigma(\sigma)$ and $x_{1}$ in $N_{x_{0}, v}$ such that $N=\left\{x+u(x) \mid x \in N_{x_{0}, v}\right\}$ is the parallel manifold of $N_{x_{0}, v}$ in $\zeta(\sigma)$ through $y=x_{1}+u\left(x_{1}\right)$. By (iv), $u\left(x_{1}\right) \in \nu(M)_{x_{1}}$, hence $y \in W \cdot x_{1}$ and $\nu(M)_{x_{1}}=\nu(M)_{y}$. Since $x_{1} \in N_{x_{0}, v},\left(x_{1}-\tilde{x}_{0}\right) \in \nu(M)_{x_{1}}$, so $\tilde{x}_{0} \in \nu(M)_{x_{1}}=\nu(M)_{y}$, which implies that $\left(\tilde{x}_{0}-y\right) \in \nu(M)_{y}$. Then (vi) follows from the fact that $y \in W \cdot x_{1}$ and $x_{1} \in N_{x_{0}, v}$. q.e.d.

Münzner showed that the number $p$ of distinct principal curvatures of an isoparametric hypersurface $M^{n}$ in $S^{n+1}$ must be $1,2,3,4$ or 6 . Viewing $M^{n}$ as a rank 2 isoparametric submanifold in $\mathbb{R}^{n+2}$ we can restate his result as follows:

2.2. Theorem [35]. If $M^{n} \subset S^{n+1} \subset \mathbb{R}^{n+2}$ is isoparametric, then the angle between any two distinct reflection lines of the associated Coxeter group $W$ on $\mathbb{R}^{2}$ is multiple of $\pi / p$, where $p=1,2,3,4$, or 6 . Equivalently, $W$ must be one of the following Coxeter groups: $A_{1}, A_{1} \times A_{1}, A_{2}, B_{2}$, or $G_{2}$.

A Coxeter group $W$ is called crystallographic (or a Weyl group) if the angle between any two of its reflection hyperplanes is $\pi / p$, where $p=1,2,3,4$, or 6 . So combining 2.1 and 2.2 we have:

2.3. Corollary. The Coxeter group associated to an isoparametric submanifold is crystallographic. 
Using 1.13 we have

2.4. Corollary. The associated Coxeter group of an irreducible isoparametric submanifold must be one of $A_{k}(k \geq 1), B_{k}(k \geq 2), D_{k}(k \geq 4), E_{6}$, $E_{7}, E_{8}, F_{4}$ or $G_{2}$.

Next we give some restrictions on the possible multiplicities $m_{i}$ for various Weyl groups. The first result of this type, proved by É. Cartan, can be formulated in our setting as follows:

2.5. Theorem (Cartan). The only possible marked Dynkin diagram for an isoparametric submanifold with $A_{2}$ as the associated Coxeter group is

$$
\underset{0}{m} \quad m
$$

where $m \in\{1,2,4,8\}$.

Using the delicate cohomology theory developed by Münzner for isoparametric hypersurfaces in spheres, Münzner and Abresch obtained restrictions on the $m_{i}$ 's for the case of $B_{2}$ and $G_{2}$.

2.6. Theorem [35], [1]. Suppose $M^{n} \subset \mathbb{R}^{n+2}$ is isoparametric. Then

(i) if

$$
\stackrel{m_{1} \quad m_{2}}{\stackrel{m_{0}}{\circ}}, \quad m_{1} \leq m_{2}
$$

is the marked Dynkin diagram of $M$, then $\left(m_{1}, m_{2}\right)$ must satisfy one of the following conditions:

(a) $2^{k}$ divides $\left(m_{1}+m_{2}+1\right)$, where $2^{k}=\min \left\{2^{\sigma} \mid m_{1}<2^{\sigma}, \sigma \in \mathbb{N}\right\}$,

(b) if $m_{1}$ is a power of 2 , then $2 m_{1}$ divides $\left(m_{2}+1\right)$ or $3 m_{1}=2\left(m_{2}+1\right)$, (ii) if

$$
\underset{m_{1}}{\stackrel{m_{2}}{=}}
$$

is the marked Dynkin diagram of $M$, then $m_{1}=m_{2} \in\{1,2\}$.

Suppose $M^{n} \subset \mathbb{R}^{n+k}$ is full and isoparametric with Weyl group $W$, $\left\{l_{1}\left(x_{0}\right), \cdots, l_{k}\left(x_{0}\right)\right\}$; is a simple root system for $W, \Delta$ is the Weyl chamber of $W$ in $\nu(M)_{x_{0}}$ containing $x_{0}$, and $\partial \Delta \subset \bigcup\left\{l_{j}\left(x_{0}\right) \mid 1 \leq j \leq k\right\}$. Let $\sigma$ denote the $(k-2)$-simplex of $\Delta$ determined by the intersection of two hyperplanes $l_{i}\left(x_{0}\right)$ and $l_{j}\left(x_{0}\right), i \neq j$. Then by 2.1 (ii) $N_{x_{0}, \sigma}$ is an isoparametric submanifold of rank two with $W_{\sigma}$ as the associated Weyl group, and $l_{i}\left(x_{0}\right) \cap\left(x_{0}+\xi\left(x_{0}, \sigma\right)\right), l_{j}\left(x_{0}\right) \cap\left(x_{0}+\xi\left(x_{0}, \sigma\right)\right)$ form a simple root system for $W_{\sigma}$ with multiplicities $m_{i}$ and $m_{j}$. Then $2.1,2.5$, and 2.6 will give us some immediate restrictions on the $m_{j}$ 's. To obtain further such restrictions we need the following cohomology theorem, which is a direct algebraic consequence of Theorem 7.14.

2.7. Theorem. Suppose $M^{n} \subset \mathbb{R}^{n+3}$ is isoparametric with the marked Dynkin diagram

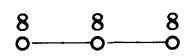


and $l_{1}\left(x_{0}\right), l_{2}\left(x_{0}\right), l_{3}\left(x_{0}\right)$ form a simple root system for $A_{3}$ such that the angle between $l_{1}$ and $l_{2}$ is $\pi / 3$. Let $\sigma$ be a 1-simplex contained in $l_{1}\left(x_{0}\right) \cap l_{2}\left(x_{0}\right)$, and let $q \in \sigma$. Then the parallel manifold $M_{q}$ to $M$ through $q$ is simply connected and its cohomology ring is

$$
H^{*}\left(M_{q}, \mathbb{Z}_{2}\right)=\mathbb{Z}_{2}[\alpha] /\left(\alpha^{4}\right), \quad \operatorname{deg}(\alpha)=8
$$

However there is the following theorem of Toda:

2.8. Theorem [43]. There is no simply connected topological space $X$ such that $H^{*}\left(X, \mathbb{Z}_{2}\right)=\mathbb{Z}_{2}[\alpha] /\left(\alpha^{4}\right)$ unless the degree of $\alpha$ is 2 or 4 .

2.9. Corollary. There is no isoparametric submanifold with the marked Dynkin diagram

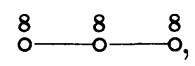

and hence there is no isoparametric submanifold whose marked Dynkin diagram contains a subdiagram of the above form.

Then it follows from 2.1, 2.4, 2.5, 2.6, and 2.9 that we have:

2.10. Theorem. The possible marked Dynkin diagrams of an irreducible isoparametric submanifold of codimension $k \geq 3$ are as follows:

$$
\begin{aligned}
& A_{k}: \stackrel{m}{\circ} \stackrel{m}{\circ} \ldots \stackrel{m}{\circ} \stackrel{m}{\circ} \quad m \in\{1,2,4\} \\
& B_{k}: \stackrel{m_{1}}{\stackrel{m_{1}}{m_{0}} . m_{1} \quad m_{2}} \quad m_{1}=1, m_{2} \text { is arbitrary } \\
& m_{1}=2, m_{2}=2 \text {, or } 2 r+1(r \geq 0) \\
& m_{1}=4, m_{2}=1,5 \text {, or } 4 r+3(r \geq 0) \\
& k=3, m_{1}=8, m_{2}=1,3,7,11 \text {, or } \\
& 8 r+7(r \geq 0)
\end{aligned}
$$

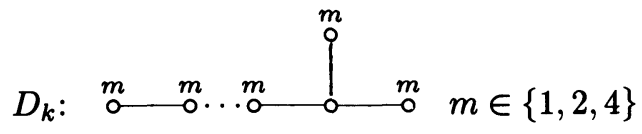

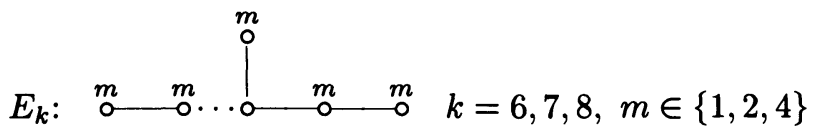

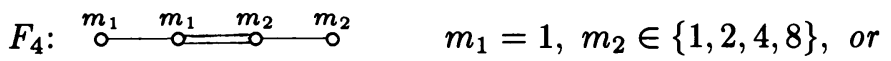

$$
\begin{aligned}
& m_{1}=m_{2}=2 \text {. }
\end{aligned}
$$

2.11. Corollary. If $M$ is irreducible, $k \geq 2$, and all the $m_{j}$ 's are even, then $m_{1}=m_{2} \in\{2,4,8\}$. 


\section{Marked Dynkin diagrams of the homogeneous isoparametric submanifolds}

The orbit geometry of the adjoint representation of a compact Lie group $G$ on its Lie algebra $\mathfrak{g}$ plays a central role in Lie Theory. The key to the understanding of this orbital geometry is the maximal torus theorem of É. Cartan. Let $T$ be a maximal torus of $G, \mathfrak{t}$ its Lie algebra (a Cartan subalgebra) and $W=N(T) / T$ the Weyl group of $G$. Then (i) the principal orbit type is $G / T$, (ii) $\mathfrak{t}$ is the fixed point set of $T$ in $\mathfrak{g}$, and $\mathfrak{t}$ intersects every $G$-orbit orthogonally, (iii) $\mathfrak{g} / G \approx \mathrm{t} / W$ and $(W, \mathfrak{t})$ is a crystallographic group. In the setting of symmetric spaces, the above adjoint representations are special cases of the isotropy representations of symmetric spaces. Let $M=G / K$ be a symmetric space, $\mathfrak{g}$ and $\mathfrak{K}$ the Lie algebras of $G$ and $K$ respectively, and $\mathfrak{P}$ the orthogonal complement of $\mathfrak{K}$ in $\mathfrak{g}$. If $\mathfrak{a}$ is a maximal abelian subalgebra of $\mathfrak{g}$ contained in $\mathfrak{P}$, then as was already known to Cartan: (i) $\mathfrak{a}$ intersects all $K$-orbits in $\mathfrak{P}$ orthogonally, (ii) there is a crystallographic group $W=N(\mathfrak{a}) / Z(\mathfrak{a})$, and $\mathfrak{P} / K \approx \mathfrak{a} / W$. These principal $K$-orbits in $\mathfrak{P}$ are isoparametric and in fact the only homogeneous isoparametric submanifolds (see 1.13). The general isoparametric foliation (see 1.9) is a geometrization of these orbital foliations. As noted in 1.14 there are many examples of non-orbital isoparametric foliations in the rank 2 case [37], [23]. Nevertheless the above orbital foliations of $(K, \mathfrak{P})$ provide the great majority of known examples and therefore it is useful to have explicit knowledge of their geometric characteristics, both for the study of the geometry of isoparametric foliations and for various specific applications of such foliations [24], [26], [27], [28], [41].

From the well-known classification of symmetric spaces [25], it is easy to write down explicitly the isotropy representation $(K, \mathfrak{P})$ of a symmetric space $G / K$. Using 1.13 , we note that the Weyl group of the principal $K$-orbits, as an isoparametric submanifold, is just the Weyl group of the symmetric space $G / K$, and the multiplicities are the differences in dimensions between a principal orbit and subprincipal orbits in $(K, \mathfrak{P})$. Hence it is not difficult to obtain the marked Dynkin diagram for these homogeneous isoparametric submanifolds. Below we will write down the results of these calculations, assuming $k \geq 2$. For convenience we divide the pairs $(G, K)$ into five families, which we discuss separately.

3.1. Adjoint orbits. For the adjoint representation of a compact connected Lie group, $(G, \mathfrak{g})$, all multiplicities are equal to 2 , the principal orbit type is the flag manifold $G / T$, and the collection of focal manifolds are exactly the homogeneous spaces (partial flags) $G / U$, where $U$ runs through all conjugacy classes of connected, maximal rank subgroups of $G$ with nondiscrete center. Let $G \rightarrow E G \rightarrow B G$ be the universal $G$-bundle and $G / U \rightarrow B U \rightarrow B G$ 
be the associated $G / U$-bundle. Then one has the following well-known results of A. Borel [3] on the rational cohomology of classifying spaces and homogeneous spaces:

(i) $H^{*}(B G) \approx H^{*}(B T)^{W(G)}$, the ring of $W(G)$-invariant polynomials, which is again a free algebra.

(ii) $H^{*}(G / U) \approx H^{*}(B U) /\left\langle H^{+}(B G)\right\rangle \approx H^{*}(B T)^{W(U)} /\left\langle H^{+}(B T)^{W(G)}\right\rangle$, where $\left\langle H^{+}(B G)\right\rangle$ and $\left\langle H^{+}(B T)^{W(G)}\right\rangle$ denote the ideals generated by homogeneous elements of positive degree in $H^{*}(B G) \approx H^{*}(B T)^{W(G)}$. One of our goals in this paper is a generalization of these results of Borel to include the case of the rational cohomology of leaves of isoparametric foliations with even multiplicities (cf. 6.12 and 6.14). However our technique for proving these results comes from Riemannian geometry, and is much closer to that of Bott and Samelson [5], than to that of Borel.

3.2. Uniform multiplicity 1 . To each simple compact Lie algebra $\mathfrak{g}$, there exists a unique conjugacy class of involutive automorphisms of $\mathfrak{g}$ which maps each root $\alpha$ to $-\alpha$. The corresponding symmetric space $G / K$ has the same rank and Weyl group as $G$. Moreover

$$
\operatorname{dim} K=\frac{1}{2}(\operatorname{dim} G-\operatorname{rank} G), \operatorname{dim}(\mathfrak{P})=\frac{1}{2}(\operatorname{dim} G+\operatorname{rank} G),
$$

and the principal orbit type of $(K, \mathfrak{P})$ is $K / Z_{2}^{k}$ with multiplicities uniformly equal to 1 . We list them as follows:

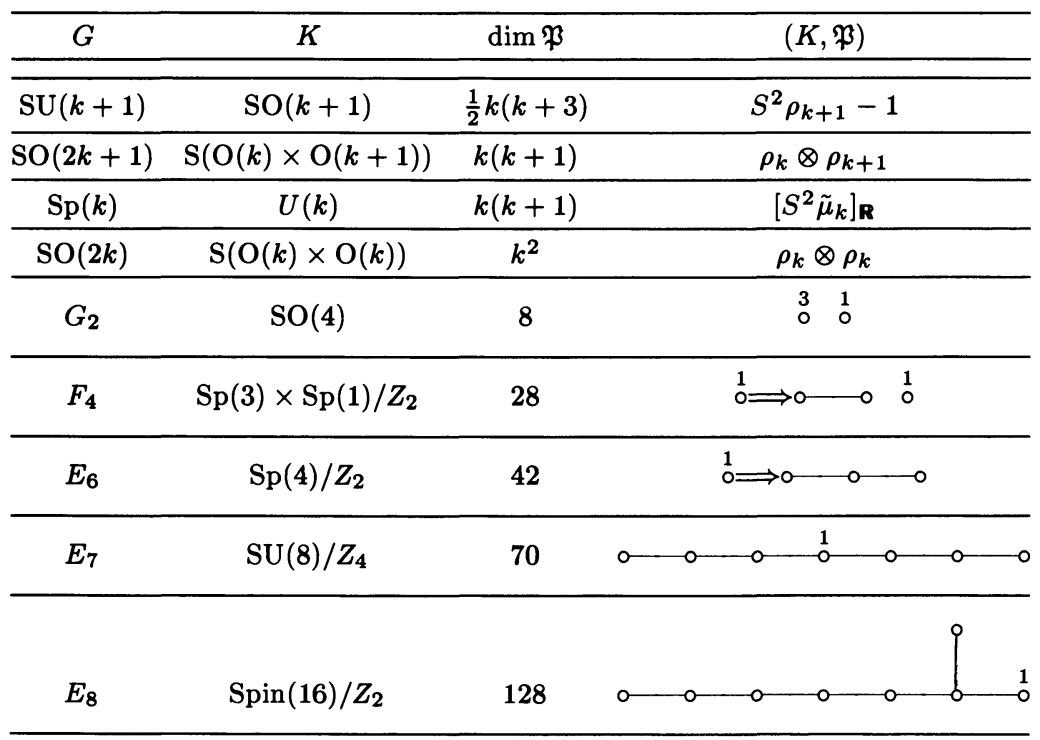


3.3. $A_{k}$-type. There are the following two additional cases of orbital isoparametric foliations with Weyl groups of type $A_{k}$ :

\begin{tabular}{|c|c|c|c|}
\hline$G$ & $K$ & $\operatorname{dim} \mathfrak{P}$ & $(K, \mathfrak{P})$ \\
\hline $\mathrm{SU}(2 k+2)$ & $\operatorname{Sp}(k+1)$ & $2 k^{2}+3 k$ & 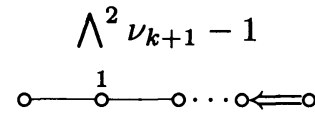 \\
\hline$E_{6}$ & $F_{4}$ & 26 & $1-0 \Longleftarrow 0$ \\
\hline
\end{tabular}

The marked Dynkin diagrams of these two cases are respectively:
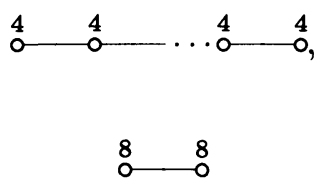

The principal orbit type for the first case is $\operatorname{Sp}(k+1) / \operatorname{Sp}(1)^{k+1}$ and for the second is $F_{4} / \operatorname{Spin}(8)$.

3.4. $F_{4}$-type. There are the following three additional cases of orbital isoparametric foliations with Weyl group of type $F_{4}$ :

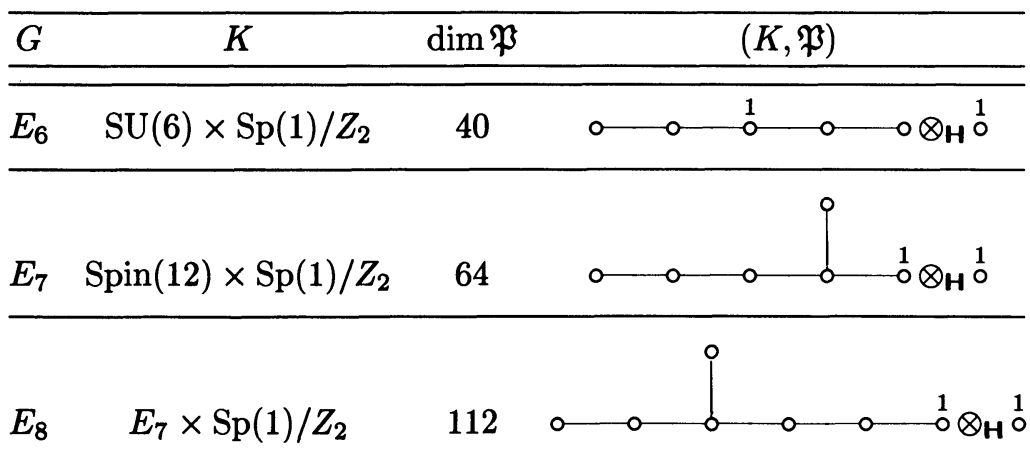

where $\otimes_{\mathbf{H}}$ denotes tensoring over the quaternions. The marked Dynkin diagrams of these three cases are respectively:

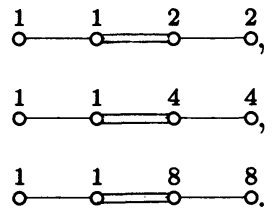


And the principal isotropy subgroups $H$ of the above representations $(K, \mathfrak{P})$ are respectively:

\begin{tabular}{cc}
\hline$K$ & $H$ \\
\hline \hline $\mathrm{SU}(6) \times \mathrm{Sp}(1) / Z_{2}$ & $Z_{2}^{2} \times T^{2}$ \\
\hline $\mathrm{Spin}(12) \times \mathrm{Sp}(1) / Z_{2}$ & $Z_{2}^{2} \times \mathrm{Sp}(1)^{3}$ \\
\hline$E_{7} \times \mathrm{Sp}(1) / Z_{2}$ & $Z_{2}^{2} \times \operatorname{Spin}(8)$ \\
\hline
\end{tabular}

3.5. $B_{k}$-type. Finally we list all the additional cases of orbital isoparametric foliations whose Weyl groups are of type $B_{k}$ with $k \geq 6$ :

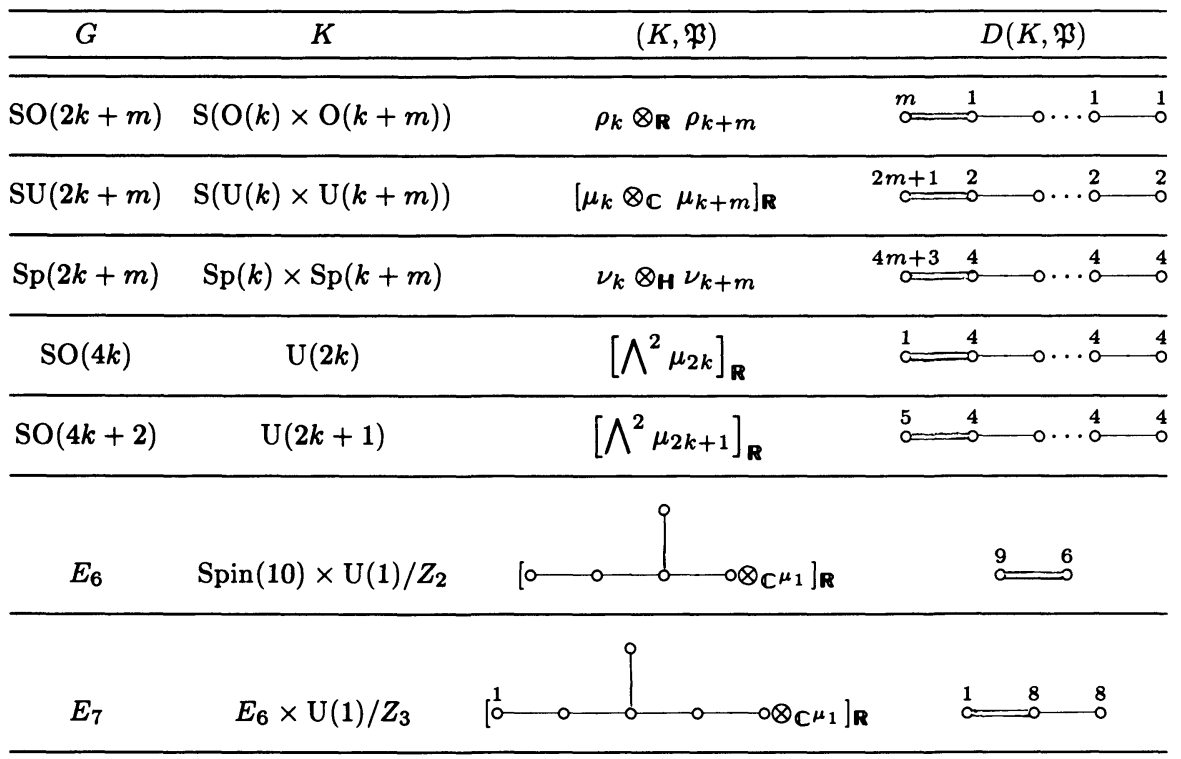

where $D(K, \mathfrak{P})$ denotes the marked Dynkin diagram for the principal $K$-orbit, $\rho_{k}=\left(\mathrm{O}(k), \mathbb{R}^{k}\right), \mu_{k}=\left(\mathrm{U}(k), \mathbb{C}^{k}\right)$ and $\nu_{k}=\left(\mathrm{Sp}(k), \mathrm{H}^{k}\right)$ are respectively the standard representations of $\mathrm{O}(k), \mathrm{U}(k)$ and $\mathrm{Sp}(k),[*]_{\mathbf{R}}$ denotes the underlying real representation of the complex representation $*$, and $\bigwedge^{2}$ and $S^{2}$ denote respectively the second exterior and second symmetric products.

3.6. Open problems. If we compare the above results on orbital isoparametric foliations with the restrictions of Theorem 2.10 on possible multiplicities of irreducible isoparametric submanifolds of rank $k \geq 3$, it is natural to pose the following problems: 
Problem 1. Is it possible to have an isoparametric foliation on $\mathbb{R}^{52}$ whose marked Dynkin diagram is of type $D_{4}$ and with all multiplicities uniformly equal to 4 ?

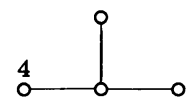

(It would be interesting if such an example does exist, however we expect that most likely it does not. Of course a negative answer to this problem would also imply the nonexistence of marked Dynkin diagrams with uniform multiplicity 4 of $D_{k}$-type, $k>5$ or $E_{k}$-type, $k=6,7,8$.)

Problem 2. Is it possible to have an isoparametric foliation whose marked Dynkin diagram is of the type

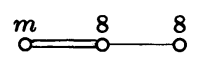

with $m>1$ ?

Problem 3. Let $M^{n} \subset \mathbb{R}^{n+k}$ be an irreducible isoparametric submanifold with uniform multiplicities. Is it necessarily homogeneous, i.e., is the associated isoparametric foliation orbital?

If the answer to Problem 3 is affirmative and if the answers to Problems 1 and 2 are both negative, then the remaining fundamental problem would be:

Problem 4. Are the examples of nonhomogeneous irreducible isoparametric submanifolds of rank $k \geq 3$ ?

So far, all the known examples of nonhomogeneous irreducible isoparametric submanifolds are of $\operatorname{rank} k=2(1.14,[23],[37])$.

\section{Morse theory}

Morse theory relates the homology, homotopy, and even the diffeomorphism type of a smooth manifold $\mathbf{X}$ to the critical point structure of certain real valued functions $f: \mathbf{X} \rightarrow \mathbb{R}$ and it will be our basic tool for studying the homology structure of isoparametric submanifolds. In this section we will review briefly the basic concepts of Morse theory, develop appropriate notation, and recall, mainly without proof, the fundamental theorems (full details can be found in [34] or [39]). Finally we shall cover some more advanced topics of Morse theory that we will need later. These are mainly results of Morse [6] and Bott and Samelson [5], but since they do not seem to be covered adequately in the generality we need, we will give an exposition with reasonably complete proofs.

$\mathbf{X}$ will denote a smooth, compact, Riemannian manifold. The inner product of $u, v \in T \mathbf{X}_{x}$ will be denoted by $\langle u, v\rangle . f: \mathbf{X} \rightarrow \mathbb{R}$ will denote a smooth real valued function and $\nabla f$ its gradient field. A point $x$ is called a critical point 
of $f$ if $d f_{x}=0$, or equivalently if $\nabla f_{x}=0$; other points of $\mathbf{X}$ are called regular points of $f$. A real number $a$ is called a regular value of $f$ if $f^{-1}(a)$ consists entirely of regular points, and otherwise a critical value of $f$. We write $\mathbf{X}^{a}$ for the set $\{x \in \mathbf{X} \mid f(x) \leq a\}$. The set $f^{-1}(a)$ will be referred to as the level $a$ (of $f$ ). If $x_{0}$ is a critical point and $u, v$ are two smooth vector fields on $\mathbf{X}$ near $x_{0}$ then

$$
u_{x_{0}}(v f)-v_{x_{0}}(u f)=d f_{x_{0}}([u, v])=0 .
$$

Since $u_{x_{0}}(v f)$ depends on $u$ only through its value at $x_{0}$ while $v_{x_{0}}(u f)$ depends on $v$ only through its value at $x_{0}$, we have:

4.1. Proposition and Definition. At a critical point $x_{0}$ of $f$ there is a well-defined symmetric bilinear form $\operatorname{Hess}(f)_{x_{0}}$ on $T \mathbf{X}_{x_{0}}$ such that if $u$ and $v$ are smooth vector fields near $x_{0}$ then $\operatorname{Hess}(f)_{x_{0}}(u, v)=u_{x_{0}}(v f)$. We define a self-adjoint operator hess $(f)_{x_{0}}$ on $T \mathbf{X}_{x_{0}}$ by

$$
\operatorname{Hess}(f)_{x_{0}}(u, v)=\left\langle\operatorname{hess}(f)_{x_{0}} u, v\right\rangle
$$

At a critical point $x_{0}$ of $f$ the nullity of $f$ at $x_{0}$ is defined as the dimension of the kernel of hess $(f)_{x_{0}}$, and $\operatorname{ind}(f)_{x_{0}}$, the index of $f$ at $x_{0}$, is defined to be the maximum dimension of a subspace on which $\operatorname{Hess}(f)_{x_{0}}$ is negative definite, or equivalently as the sum of the dimensions of the eigenspaces of hess $(f)_{x_{0}}$ corresponding to negative eigenvalues. The critical point $x_{0}$ is called nondegenerate if $\operatorname{Hess}(f)_{x_{0}}$ is a nondegenerate quadratic form, i.e., if hess $(f)_{x_{0}}$ does not have zero in its spectrum.

For our purposes a chart at $x_{0}$ will mean a diffeomorphism $\varphi$ of a neighborhood $U$ of the origin in $T \mathbf{X}_{x_{0}}$ onto a neighborhood of $x_{0}$ in $\mathbf{X}$ mapping 0 to $x_{0}$. If $x_{0}$ is a regular point of $f$ then by the implicit function theorem we can choose $\varphi$ so that $f \circ \varphi(u)=f\left(x_{0}\right)+d f_{x_{0}}(u)$; i.e., so that $f-f\left(x_{0}\right)$ is linear in an appropriate coordinate system. It follows that if $a$ is a regular value of $f$ then $\mathbf{X}^{a}$ is a smooth submanifold with boundary, whose boundary is just $f^{-1}(a)$, the level $a$. The starting point of Morse theory is an analogous canonical form theorem, the Morse Lemma, in the neighborhood of a nondegenerate critical point $x_{0}$; namely in this case the chart $\varphi$ can be chosen so that $f \circ \varphi-f\left(x_{0}\right)$ is quadratic, so the second order Taylor expansion has no remainder. More precisely the Morse Lemma states the existence of a chart $\varphi$ at $x_{0}$ such that

$$
f \circ \varphi(u)-f\left(x_{0}\right)=\frac{1}{2} \operatorname{Hess}(f)_{x_{0}}(u, u)=\langle A u, u\rangle,
$$

where $A=\frac{1}{2}$ hess $(f)_{x_{0}}$. Since zero is not in the spectrum of $A$ we can form the positive operator $|A|$, its inverse $|A|^{-1}$, and positive square root $|A|^{1 / 2}$, 
and clearly

$$
\begin{aligned}
\langle A u, u\rangle & =\left\langle|A|^{-1} A|A|^{1 / 2} u,|A|^{1 / 2} u\right\rangle \\
& =\left\langle\operatorname{sgn}(A) \psi^{-1} u, \psi^{-1} u\right\rangle,
\end{aligned}
$$

where $\operatorname{sgn}(A)=|A|^{-1} A$ and $\psi$ is the linear isomorphism $|A|^{-1 / 2}$ of $T \mathbf{X}_{x_{0}}$. Thus we have

$$
f \circ \varphi^{*}(u)-f\left(x_{0}\right)=\langle\operatorname{sgn}(a) u, u\rangle,
$$

where $\varphi^{*}$ is the chart $\varphi \circ \psi$. Now $\operatorname{sgn}(A)$ is just equal to $P^{+}-P^{-}$where $P^{ \pm}$ are the orthogonal projections of $T \mathbf{X}_{x_{0}}$ on the positive and negative spaces of $f$ at $x_{0}$ (i.e., the direct sum of the positive (resp. negative) eigenspaces of hess $(f)_{x_{0}}$ ) and we get the following more convenient form of the Morse Lemma:

4.2. Morse Lemma. If $x_{0}$ is a nondegenerate critical point of $f: \mathbf{X} \rightarrow \mathbb{R}$ and $P^{ \pm}$are the projections on the positive and negative subspaces of $f$ at $x_{0}$ then there exists a so-called Morse chart $\varphi$ for $f$ at $x_{0}$, for which

$$
f \circ \varphi(u)-f\left(x_{0}\right)=\left\|P^{+} u\right\|^{2}-\left\|P^{-} u\right\|^{2} .
$$

4.3. Corollary. A nondegenerate critical point is isolated in the set of all critical points.

We call $f: \mathbf{X} \rightarrow \mathbb{R}$ nondegenerate or a Morse function if all of its critical points are nondegenerate. Let us denote by $C(f)$ the closed and hence compact set of critical points of $f$. Then, by the corollary, if $f$ is a Morse function, $C(f)$ is also discrete and hence is finite.

We now introduce some further fixed notation. Suppose $x_{0}$ is a nondegenerate critical point of $f$ of index $k$ at the level $c$ and let $E^{k}$ denote the negative subspace of $f$ at $x_{0}$. Fix a Morse chart $\varphi: \mathcal{U} \rightarrow \mathbf{X}$ at $x_{0}$, say with $\mathcal{U}=\left\{v \in T \mathbf{X}_{x_{0}} \mid\|v\|^{2}<\delta\right\}$. Then for $\varepsilon<\delta$ we define $D^{k}(\varepsilon)$ to be the disk of radius $\sqrt{\varepsilon}$ in $E^{k}$ and $\partial D^{k}(\varepsilon)$ its boundary $k-1$ sphere. We put $e^{k}(\varepsilon)=\varphi\left(D^{k}(\varepsilon)\right), \partial e^{k}(\varepsilon)=\varphi\left(\partial D^{k}(\varepsilon)\right)$, and we call $e^{k}(\varepsilon)$ the descending cell (of radius $\sqrt{\varepsilon}$ ) at $x_{0}$. By the defining property of a Morse chart,

$$
\mathbf{X}^{c-\varepsilon} \cap e^{k}(\varepsilon)=\partial e^{k}(\varepsilon) \subseteq \partial \mathbf{X}^{c-\varepsilon}=f^{-1}(c-\varepsilon),
$$

so that $\mathbf{X}^{c-\varepsilon} \cup e^{k}(\varepsilon)$ is homeomorphic to the adjunction space $\mathbf{X}^{c-\varepsilon} \cup_{\varphi}$ $D^{k}(\varepsilon)$. As an immediate consequence of these definitions and excision we have homology isomorphisms (for any coefficients)

$$
\begin{aligned}
H_{*}\left(E^{K}, E^{k}-\{0\}\right) & \approx H_{*}\left(D^{k}(\varepsilon), \partial D^{k}(\varepsilon), \partial D^{k}(\varepsilon)\right) \approx H_{*}\left(e^{k}(\varepsilon), \partial e^{k}(\varepsilon)\right) \\
& \approx H_{*}\left(\mathbf{X}^{c-\varepsilon} \cup e^{k}(\varepsilon), \mathbf{X}^{c-\varepsilon}\right) .
\end{aligned}
$$

Thus $H_{j}\left(\mathbf{X}^{c-\varepsilon} \cup e^{k}(\varepsilon), \mathbf{X}^{c-\varepsilon}\right)$ is zero for $j \neq k$ and is the coefficient ring when $j=k$, and in the latter case a choice of generator corresponds to a choice of orientation for the Euclidean space $E^{k}$. 
A diffeotopy of $\mathbf{X}$ is a one-parameter family $\left\{\varphi_{t}\right\}$ of diffeomorphisms of $\mathbf{X}, 0 \leq t \leq 1$, such that $(t, x) \rightarrow \varphi_{t}(x)$ is a smooth map of $[0,1] \times \mathbf{X}$ into $\mathbf{X}$, and such that $\varphi_{t}$ is the identity for $t$ near zero and is $\varphi_{1}$ for $t$ near one. Then $\left\{\varphi_{t}\right\}$ is characterized by its time dependent vector field $\varphi^{\prime}(t, x):=$ $(\partial / \partial s)_{s=0} \varphi_{t+s}\left(\varphi_{t}^{-1}(x)\right)$, and conversely any time dependent vector field $\xi_{t}$ on $\mathbf{X}$ which vanishes near $t=0$ and $t=1$ gives rise to a uniquely determined diffeotopy by solving $\partial / \partial t\left(\varphi_{t}(x)\right)=\xi_{t}\left(\varphi_{t}(x)\right)$ with $\varphi_{0}(x)=x$. One of the basic tools of Morse theory is to construct in this way certain diffeotopies $\left\{\varphi_{t}\right\}$ with the property that $\varphi^{\prime}(t, x)=\psi(t, x)\left(-\nabla f_{\varphi(t, x)}\right)$, where $\psi \geq 0$, a condition we shall express by saying that $\varphi^{\prime}$ is positively proportional to $-\nabla f$. This has important consequences: it is equivalent to the condition that for any $x$ in $\mathbf{X}$ the curve $t \rightarrow \varphi(t, x), 0 \leq t \leq 1$, is contained in the positive time orbit of $x$ under the flow generated by $-\nabla f$, so in particular $\left\{\varphi_{t}\right\}$ will map into itself any subset $\mathbf{X}_{0}$ of $\mathbf{X}$ invariant under this positive time flow (in particular the sets $\mathbf{X}^{c}$ and any subset of $\left.C(f)\right)$. It also follows that $f(\varphi(t, x))$ is a nonincreasing function of $x$.

The following result is fundamental.

4.4. Deformation Theorem. Let $f$ be a smooth real valued function on a compact Riemannian manifold $\mathbf{X}$ and let $[a, b]$ be an interval of real numbers with $a<c<b$;

(a) If there are no critical values of $f$ in $[a, b]$ then there is a diffeomorphism $\varphi$ of $[a, b] \times f^{-1}(c)$ onto $f^{-1}([a, b])$ with $f(\varphi(t, x))=t$.

(b) If $c$ is the only critical value of $f$ in $[a, b]$ and $U$ is any neighborhood in $\mathbf{X}$ of the set $K$ of critical points of $f$ at the level $c$ then there is a diffeotopy $\left\{\varphi_{t}\right\}$ of $X$ with $\varphi^{\prime}$ positively proportional to $-\nabla f$ such that $\varphi_{1}\left(\mathbf{X}^{b}-U\right) \subseteq \mathbf{X}^{a}$. Moreover if $\varepsilon>0$ then we can suppose that $\mathrm{X}^{a-\varepsilon}$ and $\mathrm{X}^{b+\varepsilon}$ are pointwise fixed under $\varphi_{t}$. Finally the same is true with $U=\varnothing$ if $K=\varnothing$, i.e., if $c$ is not actually a critical value of $f$.

(c) If in addition to the assumptions of (b) $K$ consists of nondegenerate critical points, then $K$ is a finite set $\left\{x_{1}, \cdots, x_{r}\right\}$, say with indices $\left\{k_{1}, \cdots, k_{r}\right\}$, and then for $\varepsilon$ sufficiently small the descending cells $e^{k_{i}}$ of radius $\sqrt{\varepsilon}$ are disjoint and there is a deformation retraction of $\mathbf{X}^{b}$ onto $\mathbf{X}^{c-\varepsilon} \cup e^{k_{1}} \cup \cdots \cup e^{k_{r}}$.

Proof. See [34] and [38].

We now add some standing notational conventions. The coefficients for homology and cohomology will be a commutative ring with unit, $R$. In applications $R$ will usually either be $\mathbb{Z}$ (if everything is orientable) or $\mathbb{Z}_{2}$. Given the assumptions of $4.4(\mathrm{c})$ above we will denote by $\left[e^{k_{i}}, \partial e^{k_{i}}\right]$ the generator of $H_{k_{i}}\left(e^{k_{i}}, \partial e^{k_{i}} ; R\right)$ determined by some orientation of $e^{k(i)}$. Now according to 
4.4(c) we have an excision isomorphism

$$
\bigoplus_{i \leq r} H_{*}\left(e^{k_{i}}, \partial e^{k_{j}}\right) \approx H_{*}\left(\mathbf{X}^{c-\varepsilon} \cup \bigcup_{i \leq r} e^{k_{i}}, \mathbf{X}^{c-\varepsilon}\right)
$$

and we have a deformation retraction

$$
\mathbf{X}^{b} \approx \mathbf{X}^{c-\varepsilon} \cup \bigcup_{i \leq r} e^{k_{i}}
$$

and by $4.4(\mathrm{a})$ (or (b)) another deformation retraction $\mathrm{X}^{c-\varepsilon} \approx \mathrm{X}^{a}$. Putting these all together it follows that the inclusion map

$$
\bigcup_{i \leq r}\left(e^{k_{i}}, \partial e^{k_{i}}\right) \subseteq\left(\mathbf{X}^{c-\varepsilon} \cup \bigcup_{i \leq r} e^{k_{i}}, \mathbf{X}^{c-\varepsilon}\right) \subseteq\left(\mathbf{X}^{b}, \mathbf{X}^{a}\right)
$$

induces an isomorphism

$$
H_{*}\left(\mathbf{X}^{b}, \mathbf{X}^{a}\right) \approx \bigoplus_{i \leq r} H_{*}\left(e^{k_{i}}, \partial e^{k_{i}}\right)
$$

and we shall again denote by $\left[e^{k_{i}}, \partial e^{k_{i}}\right]$ the image in $H_{*}\left(\mathbf{X}^{b}, \mathbf{X}^{a}\right)$ of the above generators of the $H_{k_{i}}\left(e^{k_{i}}, \partial e^{k_{i}}\right)$, so we have: $R$

4.5. Corollary. With the assumptions of $4.4(\mathrm{c})$, for any coefficient ring

$$
H_{k}\left(\mathbf{X}^{b}, \mathbf{X}^{a}\right)=\bigoplus_{k_{i}=k} R\left[e^{k_{i}}, \partial e^{k_{i}}\right]
$$

Now let $x_{0}$ be a nondegenerate critical point of index $k$ at the level $c$ of $f: \mathbf{X} \rightarrow \mathbb{R}$ and let $e^{k}$ denote its descending cell of radius $\sqrt{\varepsilon}$. Following Morse and Cairns [6] we shall say that $x_{0}$ is of linking type with respect to $R$ if the relative homology class $\left[e_{k}, \partial e_{k}\right]$ in $H_{k}\left(\mathbf{X}^{c-\varepsilon} \cup e^{k}, \mathbf{X}^{c-\varepsilon} ; R\right)$ is in the kernel of

$$
\partial: H_{k}\left(\mathbf{X}^{c-\varepsilon} \cup e^{k}, \mathbf{X}^{c-\varepsilon} ; \mathbf{R}\right) \rightarrow H_{k-1}\left(\mathbf{X}^{c-\varepsilon} ; R\right)
$$

or equivalently, by exactness of the homology sequence for $\left(\mathbf{X}^{c-\varepsilon} \cup e^{k}, \mathbf{X}^{c-\varepsilon}\right)$, if $\left[e^{k}, \partial e^{k}\right]$ is in the image of the projection

$$
j_{*}: H_{k}\left(\mathbf{X}^{c-\varepsilon} \cup e^{k} ; R\right) \rightarrow H_{k}\left(\mathbf{X}^{c-\varepsilon} \cup e^{k}, \mathbf{X}^{c-\varepsilon} ; R\right) .
$$

If $\mu \in H_{k}\left(\mathbf{X}^{c-\varepsilon} \cup e^{k} ; \mathbf{R}\right)$ is such that $j_{*}(\mu)=\left[e^{k}, \partial e^{k}\right]$ we shall call $\mu$ (or any representative) a linking cycle for $x_{0}$ with respect to $R$. More generally if $\mathbf{X}^{c-\varepsilon} \cup e^{k} \subseteq \mathbf{Y} \subseteq \mathbf{X}$ and $i: \mathbf{X}^{c-\varepsilon} \cup e^{k} \rightarrow \mathbf{Y}$ is the inclusion map then the image $i_{*}(\mu)$ of $\mu$ in $H_{*}(\mathbf{Y} ; R)$ will also be called a linking cycle for $x_{0}$. Finally a Morse function $f: \mathbf{X} \rightarrow \mathbf{R}$ is said to be of linking type with respect to $R$ if each of its critical points is. 
4.6. Theorem. Assume that $c \in(a, b)$ is the only critical value of $f: \mathbf{X} \rightarrow$ $\mathbb{R}$ in the interval $[a, b]$ and that the critical points $\left\{x_{1}, \cdots, x_{r}\right\}$ of $f$ at the level $c$ are all nondegenerate and of linking type with respect to $R$. If $x_{i}$ has index $k_{i}$ and $\mu_{i} \in H_{k_{i}}\left(\mathbf{X}^{b} ; R\right)$ is a linking cycle for $x_{i}$ with respect to $R$ then

$$
H_{*}\left(\mathbf{X}^{b} ; R\right)=H_{*}\left(\mathbf{X}^{a} ; R\right) \oplus \bigoplus_{i=1}^{r} R \mu_{i}
$$

Proof. Recall from Corollary 4.5 that $H_{*}\left(\mathbf{X}^{b}, \mathbf{X}^{a} ; R\right)$ is the direct sum

$$
\bigoplus R\left[e^{k_{i}}, \partial e^{k_{i}}\right]
$$

where $e^{k_{i}}$ is the descending cell of $f$ at $x_{i}$ of sufficiently small radius. Since each $x_{i}$ is of linking type, each $\left[e^{k_{i}}, \partial e^{k_{i}}\right]$ is in the kernel of $\partial: H_{*}\left(\mathbf{X}^{b}, \mathbf{X}^{a} ; R\right) \rightarrow$ $H_{*}\left(\mathbf{X}^{a} ; R\right)$, so that $\partial=0$ and the homology exact sequence of the pair $\left(\mathbf{X}^{b}, \mathbf{X}^{a}\right)$ becomes

$$
0 \rightarrow H_{*}\left(\mathbf{X}^{a} ; R\right) \rightarrow H_{*}\left(\mathbf{X}^{b} ; \uparrow R\right) \rightarrow \bigoplus R\left[e^{k_{i}}, \partial e^{k_{i}}\right] \rightarrow 0
$$

and moreover, by definition of linking cycle, the map

$$
\sum_{i} r_{i}\left[e^{k_{i}}, \partial e^{k_{i}}\right] \rightarrow \sum_{i} r_{i} \mu_{i}
$$

clearly splits this exact sequence.

4.7. Corollary. If $f: \mathbf{X} \rightarrow \mathbb{R}$ is a Morse function of linking type with respect to $R$ and for each $x$ in $C(f) \mu_{x}$ in $H_{*}(\mathbf{X} ; R)$ is a linking cycle for $f$ with respect to $R$, then

$$
H_{*}(\mathbf{X} ; R)=\bigoplus_{x \in C(f)} R \mu_{x} .
$$

In particular if $R$ is a field then $\operatorname{dim} H_{k}(\mathbf{X} ; R)$ is equal to the number of critical points of $f$ of index $k$.

Proof. Let $c_{0}<c_{1}<\cdots<c_{n}$ be the critical points of $f$ in increasing order and choose $a_{0}, \cdots, a_{n+1}$ with $a_{i}<c_{i}<a_{i+1}$. Then applying 4.6 with $a=a_{i}, c=c_{i}$, and $b=a_{j+1}$ (and noting that because $c_{0}$ is the minimum of $\left.f, \mathbf{X}^{a_{0}}=\varnothing\right)$, and obvious induction completes the proof.

Given a Morse function $f: \mathbf{X} \rightarrow \mathbb{R}$ we define $c_{k}(f)$ to be the number of critical points of $f$ of index $k$ and $c(f)=\sum_{k} c_{k}(f)$ to be the total number of critical points of $f$. The minimum of $c(f)$ as $f$ ranges over all Morse functions $f: \mathbf{X} \rightarrow \mathbf{R}$ is called the Morse number of $\mathbf{X}$ and is denoted by $M(\mathbf{X})$. A Morse function $f: \mathbf{X} \rightarrow \mathbb{R}$ such that $c(f)=M(f)$ is called tight.

4.8. Corollary. A Morse function $f: \mathbf{X} \rightarrow \mathbb{R}$ which is of linking type with respect to some field of coefficients $\mathbf{F}$ is tight. 
Proof. By $4.7 c_{k}(f)=\operatorname{dim} H_{k}(\mathbf{X} ; \mathbf{F})$, so $c(f)=\operatorname{dim} H(\mathbf{X} ; \mathbf{F})$ and it will suffice to show that for any other Morse function $g$ on $\mathbf{X} c_{k}(g) \geq \operatorname{dim} H_{k}(\mathbf{X} ; \mathbf{F})$. But this is precisely the statement of the weak form of the Morse inequalities [34]. q.e.d.

It is clear from the above that it would be useful to develop criteria for recognizing when a Morse function is of linking type, which means of course that we must develop methods for constructing linking cycles. One of the most powerful techniques for doing this was developed in a well-known paper of R. Bott and H. Samelson [5].

4.9. Definition. Let $x_{0}$ be a nondegenerate critical point of index $k$ of $f: \mathbf{X} \rightarrow \mathbb{R}$ and let $(\mathbf{Y}, \varphi)$ be a pair with $\mathbf{Y}$ a compact smooth $k$-manifold, and $\varphi: \mathbf{Y} \rightarrow \mathbf{X}$ a smooth map. We call $(\mathbf{Y}, \varphi)$ a Bott-Samelson cycle for $f$ at $x_{0}$ if $\tilde{f}=f \circ \varphi$ has a unique and nondegenerate maximum at $y_{0}=\varphi^{-1}\left(x_{0}\right)$. We call such a pair $R$-orientable if $\mathbf{Y}$ is orientable over $R$, in which case we denote by $\varphi_{*}([\mathbf{Y}]) \in H_{k}(\mathbf{X}, R)$ the image of the fundamental class $[\mathbf{Y}] \in H_{k}(\mathbf{Y}, R)$ determined by some $R$-orientation of $\mathbf{Y}$. The critical point $x_{0}$ is said to be of Bott-Samelson type with respect to $R$ if an $R$-orientable Bott-Samelson cycle for $f$ at $x_{0}$ exists, and a Morse function $f$ is said to be of Bott-Samelson type with respect to $R$ if each of its critical points is.

The fundamental result of Bott and Samelson, which we will prove below, is that if $(\mathbf{Y}, \varphi)$ is an $\mathbf{R}$-orientable Bott-Samelson cycle for $f$ at $x_{0}$, then $x_{0}$ is of linking type with respect to $\mathbf{R}$, and in fact $\varphi_{*}([\mathbf{Y}])$ is a linking cycle for $f$ at $x_{0}$ with respect to $R$. Put more succinctly, Bott-Samelson type implies linking type. We shall in fact prove a more general relative version (Theorem 4.19) and we next introduce some appropriate notation. $\mathbf{Y}$ will denote a compact, connected space and $\mathbf{Y}_{0}$ a closed subspace. Later we specialize to the case that $\left(\mathbf{Y}, \mathbf{Y}_{0}\right)$ is a smooth relative $k$-manifold, i.e. $\mathbf{Y}-\mathbf{Y}_{0}$ is a smooth $k$-dimensional manifold, and in the main application $\mathbf{Y}_{\mathbf{0}}=\varnothing \cdot \mathbf{X}_{\mathbf{0}}$ will denote a closed subspace of $\mathbf{X}$ invariant under the positive time flow generated by $-\nabla f$ (in applications it will be either empty, of the form $\mathbf{X}^{a}$, or a subset of the set $C(f)$ of critical points), and $\alpha$ will denote some element of the set $\left[\left(\mathbf{Y}, \mathbf{Y}_{0}\right),\left(\mathbf{X}, \mathbf{X}_{0}\right)\right]$ of homotopy classes of maps $\varphi: \mathbf{Y} \rightarrow \mathbf{X}$ such that $\varphi\left(\mathbf{Y}_{0}\right) \subseteq \mathbf{X}_{0}$. If $\varphi \in \alpha$ we write $\max (f \circ \varphi)$ for the maximum of $f$ on the image of $\varphi$ and

$$
\operatorname{minimax}(f, \alpha):=\inf \{\max (f \circ \varphi) \mid \varphi \in \alpha\} .
$$

We shall also say that $\alpha$ hangs up at the level $c$ to express the fact that $\operatorname{minimax}(f, \alpha)=c$.

4.10. Minimax Principle. If $\alpha \in\left[\left(\mathbf{Y}, \mathbf{Y}_{0}\right),\left(\mathbf{X}, \mathbf{X}_{0}\right)\right]$ hangs up at the level $c$ of $f$ then $c$ must be a critical level of $f$. 
Proof. Suppose not, so by the Deformation Theorem (4.4(b) with $U=\varnothing$ ) there is a diffeotopy $\left\{\varphi_{t}\right\}$ carrying $\mathbf{X}^{c+\varepsilon}$ into $\mathbf{X}^{c-\varepsilon}$ and satisfying $\varphi_{t}\left(\mathbf{X}_{0}\right) \subseteq$ $\mathbf{X}_{0}$. Choose $\psi \in \alpha$ with $\max (f \circ \psi)<c+\varepsilon$, i.e., with $\operatorname{im}(\psi) \subseteq \mathbf{X}^{c+\varepsilon}$. Then $\operatorname{im}\left(\varphi_{1} \circ \psi\right) \subseteq \mathbf{X}^{c-\varepsilon}$ and since $\psi\left(\mathbf{Y}_{0}\right) \subseteq \mathbf{X}_{0}, \varphi_{t}\left(\psi\left(\mathbf{Y}_{0}\right)\right) \subseteq \mathbf{X}_{0}$, and hence $\varphi_{1} \circ \psi \in \alpha$. But then $\operatorname{minimax}(f, \alpha) \leq \min \left(f \circ\left(\varphi_{1} \circ \psi\right)\right) \leq c-\varepsilon$, a contradiction.

4.11. Definition. If $\alpha \in\left[\left(\mathbf{Y}, \mathbf{Y}_{0}\right),\left(\mathbf{X}, \mathbf{X}_{0}\right)\right]$ hangs up at the level $c$ of $f$ and $S \subseteq f^{-1}(c)$, then we shall say that $\alpha$ hangs up on $S$ if for any neighborhood $U$ of $S$ in $\mathbf{X}$ there is a positive $\varepsilon$ and a representative $\varphi$ of $\alpha$ such that $\operatorname{im}(\varphi) \subseteq \mathbf{X}^{c-\varepsilon} \cup U$.

4.12. Proposition. If $\alpha$ hangs up at the level $c$ of $f$ and $K$ is the set of all critical points at the level $c$ then $\alpha$ hangs up on $K$.

Proof. Similar to 4.10 but with $K$ (and hence $U$ ) nonempty in the application of $4.4(\mathrm{~b})$.

4.13. Proposition. Let $f: \mathbf{X} \rightarrow \mathbb{R}$ be a Morse function and assume that $\alpha \in\left[\left(\mathbf{Y}, \mathbf{Y}_{0}\right),\left(\mathbf{X}, \mathbf{X}_{0}\right)\right]$ hangs up on the subset $S$ of the level $c$, where $c>$ $\max \left(f \mid \mathbf{X}_{0}\right)$. Let $\left\{x_{1}, \cdots, x_{s}\right\}=S \cap C(f)$ and let $e_{i}$ denote the descending cell of radius $\sqrt{\varepsilon}$ at $x_{i}$. Then for $\varepsilon$ sufficiently small $\alpha$ has a representative $\varphi$ with $\operatorname{im}(\varphi) \subseteq \mathbf{X}^{c-\varepsilon} \cup e_{1} \cup \cdots \cup e_{s}$. In particular $\alpha$ hangs up on $\left\{x_{1}, \cdots, x_{s}\right\}$.

Proof. For $\varepsilon$ sufficiently small we have $\mathbf{X}_{0} \subseteq \mathbf{X}^{c-\varepsilon}$ and we can choose a neighborhood $U$ of $S$ in $\mathbf{X}$ with $e_{i} \subseteq U$ and $U$ disjoint from the descending cells of critical points of $f$ not in $S$. Since $\alpha$ hangs up on $S$ we can find a representative $\varphi_{0}$ of $\alpha$ with $\operatorname{im}\left(\varphi_{0}\right) \subseteq \mathrm{X}^{c-\varepsilon} \cup U$, and by 4.4(c) we can find a deformation retraction $\psi$ of $\mathbf{X}^{c+\varepsilon}$ onto $\mathbf{X}^{c-\varepsilon} \cup E$, where $E$ is the union of the descending cells of radius $\sqrt{\varepsilon}$ at all the critical points at the level $c$. Then we can take $\varphi=\psi \circ \varphi_{0}$.

4.14. Corollary. If $\alpha$ is nontrivial (i.e. no representative is constant) then at least one of the critical points in $S$ must have positive index.

Proof. Suppose not, so that $e_{i}=x_{i}$ for $i=1, \cdots, s$, and hence $\mathrm{X}^{c-\varepsilon} \cup$ $e_{1} \cup \cdots \cup e_{s}$ is just the disjoint union of $\mathbf{X}^{c-\varepsilon}$ and the discrete set $\left\{x_{1}, \cdots, x_{s}\right\}$. Since $\mathbf{Y}$ is connected and $\operatorname{im}(\varphi) \subseteq \mathbf{X}^{c-\varepsilon}$ is impossible by choice of $c$, we must have $\operatorname{im}(\varphi) \subseteq\left\{x_{j}\right\}$ for some $i$, a contradiction. q.e.d.

Henceforth we assume that $\left(\mathbf{Y}, \mathbf{Y}_{0}\right)$ is a smooth, connected relative $k$ manifold. Moreover we assume that it is oriented over our coefficient ring $R$ (of course if $R$ is $\mathbb{Z}_{2}$ this is not an extra assumption) and we let [Y, $\left.\mathbf{Y}_{0}\right]$ denote its fundamental class in $H_{k}\left(\mathbf{Y}, \mathbf{Y}_{0} ; R\right)$. Given $\alpha$ in $\left[\left(\mathbf{Y}, \mathbf{Y}_{0}\right),\left(\mathbf{X}, \mathbf{X}_{0}\right)\right]$ we let $\alpha_{*}\left(\left[\mathbf{Y}, \mathbf{Y}_{0}\right]\right)$ in $H_{k}\left(\mathbf{X}, \mathbf{X}_{0} ; R\right)$ denote $\varphi_{*}\left(\left[\mathbf{Y}, \mathbf{Y}_{0}\right]\right)$ for any $\varphi$ in $\alpha$.

4.15. Theorem. Let $f: \mathbf{X} \rightarrow \mathbb{R}$ be a Morse function and suppose $\alpha \in$ $\left[\left(\mathbf{Y}, \mathbf{Y}_{0}\right),\left(\mathbf{X}, \mathbf{X}_{0}\right)\right]$ hangs up on the level $c$ of $f$, where $c>\max \left(f \mid \mathbf{X}_{0}\right)$. If $\left(\mathbf{Y}, \mathbf{Y}_{0}\right)$ is a smooth, connected relative $k$-manifold then in fact $\alpha$ hangs up 
on the set of those critical points $x$ of $f$ at the level $c$ where $\operatorname{ind}(f)_{x} \leq k$. If $\alpha$ is nontrivial then at least one of these critical points has positive index. In particular, if $k=1$ and $\alpha$ is nontrivial then there is at least one critical point of index 1 on the level $c$.

Proof. Let $\left\{x_{1}, \cdots, x_{s}\right\}$ be the set of critical points of $f$ at the level $c$ and let $k_{i}=\operatorname{ind}(f)_{x_{i}}$ and $e^{k_{i}}$ the descending cell of radius $\sqrt{\varepsilon}$ at $x_{i}$. We can suppose $\varepsilon$ is so small that $\mathrm{X}_{0} \subseteq \mathrm{X}^{c-2 \varepsilon}$, and by Theorem 4.13 we can find a representative $\varphi$ of $\alpha$ with $\operatorname{im}(\varphi) \subseteq \mathbf{X}^{c-\varepsilon} \cup e^{k_{1}} \cup \cdots \cup e^{k_{s}}$. It will suffice to show that given $k_{i}>k$ we can find $\tilde{\varphi}$ in $\alpha$ such that $\tilde{\varphi}$ agrees with $\varphi$ outside $\varphi^{-1}\left(e^{k_{i}}\right)$ and

$$
\operatorname{im}(\tilde{\varphi}) \subseteq \mathbf{X}^{c-\varepsilon} \cup \bigcup_{j \neq 1} e^{k_{j}}
$$

Now if $\operatorname{im}(\varphi)$ misses at least one point $z$ of $\operatorname{int}\left(e^{k_{i}}\right)$ this is obvious, for there is a deformation retraction $r: e^{k_{i}}-\{z\} \rightarrow \partial e^{k_{i}}=e^{k_{i}} \cap \mathrm{X}^{c-\varepsilon}$ and we can simply redefine $\varphi$ to be $r \circ \varphi$ in $\varphi^{-1}\left(e^{k_{i}}\right)$ to get $\tilde{\varphi}$. On the other hand if $\varphi$ is smooth in the open set $\varphi^{-1}\left(e^{k_{i}}\right)$ of the $k$-dimensional smooth manifold $\mathbf{Y}-\mathbf{Y}_{0}$, then since $k_{i}>k$, by Sard's Theorem $\operatorname{im}(\varphi) \cap\left(e^{k_{i}}-\partial e^{k_{i}}\right)$ is a set of measure zero and such a $z$ surely exists. Finally by standard elementary approximation theory $\varphi$ is anyway homotopic to a map $\tilde{\varphi}$ which agrees with $\varphi$ outside $e^{k_{i}}$ and is smooth in $e^{k_{i}}-\partial e^{k_{i}}$. The final conclusions are immediate from 4.14. q.e.d.

We will need the following two known special cases of 4.15 .

4.16. Theorem. If $f: \mathbf{X} \rightarrow \mathbb{R}$ is a Morse function and some component of $\mathbf{X}$ contains two distinct local minima, $x_{0}$ and $x_{1}$, then that component also contains a critical point of index one. In fact if $\alpha$ is a homotopy class of paths $\sigma: I \rightarrow \mathbf{X}$ with $\sigma(0)=x_{0}$ and $\sigma(1)=x_{1}$ then $\alpha$ hangs up on a critical point of index one.

Proof. Take $\mathbf{X}_{0}=\left\{x_{0}, x_{1}\right\}$ and suppose $f\left(x_{0}\right) \leq f\left(x_{1}\right)$. By the Morse Lemma we can find a neighborhood $\mathcal{U}$ of $x_{1}$ so that $f(x)>f\left(x_{1}\right)+\varepsilon$ on $\partial \mathcal{U}$. Since every $\sigma$ in $\alpha$ must meet $\partial \mathcal{U}, \max (f \circ \sigma) \geq f\left(x_{1}\right)+\varepsilon$, so

$$
\sup \left\{f(x) \mid x \in \mathbf{X}_{0}\right\}<f\left(x_{1}\right)+\varepsilon \leq \operatorname{minimax}(f, \alpha) .
$$

Taking $Y=[0,1], Y_{0}=\partial Y=\{0,1\}$, the result now follows from 4.15.

4.17. Theorem. If $\mathbf{X}$ admits a Morse function $f: \mathbf{X} \rightarrow \mathbb{R}$ having no critical points of index one then each component of $\mathbf{X}$ is simply connected.

Proof. Take $Y=S^{1}, Y_{0}=X_{0}=\varnothing$ in 4.15. If some component of $X$ were not simply connected we could choose a nontrivial free homotopy class $\alpha$ in $\left[S^{1}, \mathbf{X}\right]=\left[\left(\mathbf{Y}, \mathbf{Y}_{0}\right),\left(\mathbf{X}, \mathbf{X}_{0}\right)\right]$, and by $4.15 \alpha$ must hang up on at least one critical point of index one. 
4.18. Lemma. Let $x_{0}$ be a nondegenerate critical point of $f$ of index $k$, lying on the level $c,\left(\mathbf{Y}, \mathbf{Y}_{0}\right)$ a smooth, connected, relative $k$-manifold that is orientable over $R$, and $\varphi:\left(\mathbf{Y}, \mathbf{Y}_{0}\right) \rightarrow\left(\mathbf{X}^{c}, \mathbf{X}^{c-\varepsilon}\right)$ a map such that:

(1) $\varphi$ is smooth on $\mathbf{Y}-\mathbf{Y}_{0}$,

(2) $\operatorname{im}(\varphi) \cap f^{-1}(c)=\left\{x_{0}\right\}$,

(3) $y_{0}=\varphi^{-1}\left(x_{0}\right)$, and

(4) $\varphi$ is transversal at $y_{0}$ to the ascending cell of $f$ at $x_{0}$.

Then the element $\varphi_{*}\left(\left[\mathbf{Y}, \mathbf{Y}_{0}\right]\right)$ of $H_{k}\left(\mathbf{X}^{c}, \mathbf{X}^{c-\varepsilon} ; R\right)$ is equal to $\pm\left[e^{k}, \partial e^{k}\right]$, where $e^{k}$ is the descending cell of $f$ at $x_{0}$.

Proof. In a Morse chart for $f$ at $x_{0}$ let $E^{k}$ denote the descending subspace, $\left(E^{k}\right)^{\perp}$ the orthogonal complement of $E^{k}$, and $P$ the orthogonal projection of $T \mathbf{X}_{x_{0}}$ onto $E^{k}$. Since $\operatorname{ker}(P)=\left(E^{k}\right)^{\perp}$ and $(D \varphi)_{y_{0}}$ is transversal to $\left(E^{k}\right)^{\perp}$ by assumption (4), it follows that $P \circ(D \varphi)_{y_{0}}$ is an isomorphism of $T \mathbf{Y}_{y_{0}}$ onto $E^{k}$, so that by the inverse function theorem $P \circ \varphi$ maps a small neighborhood of $y_{0}$ in $Y$ diffeomorphically onto a neighborhood of $x_{0}$ in $e^{k}$. By a standard approximation lemma we can $C^{1}$ approximate $\varphi$ by a map $\tilde{\varphi}$ which is homotopic to $\varphi$ (rel. $\mathbf{Y}_{0}$ ), agrees with $\varphi$ outside a small neighborhood of $y_{0}$, and agrees with $P \circ \varphi$ near $y_{0}$. It then follows from excision that $\varphi_{*}\left(\left[\mathbf{Y}, \mathbf{Y}_{0}\right]\right)=\tilde{\varphi}_{*}\left(\left[\mathbf{Y}, \mathbf{Y}_{0}\right]\right)= \pm\left[e^{k}, \partial e^{k}\right]$, the sign depending on whether $P \circ(D \varphi)_{y_{0}}$ preserves or reverses orientation.

4.19. Theorem. Let $x_{0}$ be a nondegenerate critical point of $f$ of index $k$, lying on the level $c, e^{k}$ the descending cell of radius $\sqrt{\varepsilon}$ at $x_{0}$, and $\left[e^{k}, \partial e^{k}\right]$ the generator of $H_{k}\left(\mathbf{X}^{c-\varepsilon} \cup e^{k}, \mathbf{X}^{c-\varepsilon} ; R\right)$. Suppose $\left(\mathbf{Y}, \mathbf{Y}_{0}\right)$ is a smooth, connected relative $k$-manifold that is orientable over $R$ and $\varphi:\left(\mathbf{Y}, \mathbf{Y}_{0}\right) \rightarrow\left(\mathbf{X}^{c}, \mathbf{X}^{c-\varepsilon}\right)$ is smooth on $\mathbf{Y}-\mathbf{Y}_{0}$. If $\tilde{f}=f \circ \varphi$ has a unique and nondegenerate maximum at $y_{0}=\varphi^{-1}\left(x_{0}\right)$ then

$$
\alpha_{*}\left(\left[\mathbf{Y}, \mathbf{Y}_{0}\right]\right)= \pm i_{*}\left(\left[e^{k}, \partial e^{k}\right]\right)
$$

where $\alpha$ is the class of $\varphi$ in $\left[\left(\mathbf{Y}, \mathbf{Y}_{0}\right),\left(\mathbf{X}^{c}, \mathbf{X}^{c-\varepsilon}\right)\right]$ and $i$ is the inclusion of $\left(\mathbf{X}^{c-\varepsilon} \cup e^{k}, \mathbf{X}^{c-\varepsilon}\right)$ in $\left(\mathbf{X}^{c}, \mathbf{X}^{c-\varepsilon}\right)$. In particular if $\mathbf{Y}_{0}$ is empty then $\alpha_{*}([\mathbf{Y}]) \in$ $H_{k}\left(\mathbf{X}^{c} ; R\right)$ is a linking cycle for $x_{0}$ with respect to $R$.

Proof. Conditions (1)-(3) of the lemma are trivially satisfied and (4) follows easily from the nondegeneracy of $\tilde{f}$ at $y_{0}$.

4.20. Theorem. A Morse function $f: \mathbf{X} \rightarrow \mathbb{R}$ which is of Bott-Samelson type with respect to a ring $R$ is also of linking type with respect to $R$. In fact if for each critical point $x$ of $f,\left(\mathbf{Y}^{x}, \varphi^{x}\right)$ is an R-orientable Bott-Samelson cycle for $f$ at $x$ then $\varphi_{*}^{x}\left(\left[\mathbf{Y}^{x}\right]\right)$ is a linking cycle for $f$ at $x$ with respect to $R$, and hence the set of these $\varphi_{*}^{x}\left(\left[\mathbf{Y}^{x}\right]\right)$ forms an $R$-module basis for $H_{*}(\mathbf{X} ; R)$. For the case $R=\mathbb{Z}$ it follows that $H_{*}(\mathbf{X})=H_{*}(\mathbf{X} ; \mathbb{Z})$ is a free abelian group with one generator $\varphi_{*}^{x}\left(\left[\mathbf{Y}^{x}\right]\right)$ for each critical point $x$ of $f$, and in particular 
it has no torsion. Finally if $f$ has no critical point of index one then each component of $\mathbf{X}$ is simply-connected.

Proof. Recalling the definition of a Bott-Samelson cycle (4.9), this is an easy consequence of the preceding theorem and Theorems 4.7 and 4.17. q.e.d.

Our main technique for studying the topology of an isoparametric submanifold $\mathcal{M}$ of $\mathbb{R}^{N}$ will be to apply the above results to the function $f^{a}: \mathcal{M} \rightarrow \mathbb{R}$, where $f^{a}(x)=\frac{1}{2}\|x-a\|^{2}$. The beautiful Index Theorem of Morse states that this is a Morse function as long as $a$ is not a focal point of $\mathcal{M}$. The Index Theorem moreover gives an algorithm for locating the critical points of $f^{a}$ (and computing the index at each one) in terms of the basic geometric data of the submanifold-namely its first and second fundamental forms or equivalently, its focal point structure. Below we give a brief exposition of this theorem in a form convenient for our application.

In the following $\mathcal{M}$ will denote a smooth submanifold of $\mathbb{R}^{N}$ and $T \mathcal{M}$ and $\nu(\mathcal{M})$ its tangent and normal bundles, both regarded as submanifolds of $\mathbb{R}^{N} \times$ $\mathbb{R}^{N}$. The Gauss map of $\mathcal{M}$ is the map $P: \mathcal{M} \rightarrow L\left(\mathbb{R}^{N}, \mathbb{R}^{N}\right)$ where $L\left(\mathbb{R}^{N}, \mathbb{R}^{N}\right)$ denotes the vector space of linear operators on $\mathbb{R}^{N}$ and $P_{x}$ is orthogonal projection of $\mathbb{R}^{N}$ into $T \mathcal{M}_{x}$. The connections induced on $T \mathcal{M}$ and $\nu(\mathcal{M})$ from the flat connection for $\mathcal{M} \times \mathbb{R}^{N}$ will be denoted respectively by $D^{T}$ and $D^{\nu}$; explicitly, given $\xi$ in $T \mathcal{M}_{x}$, a smooth curve $\sigma:(-\varepsilon, \varepsilon) \rightarrow \mathcal{M}$ with $\sigma^{\prime}(0)=\xi$ and a smooth section $u(t)$ of $T \mathcal{M}$ (resp. $\nu(\mathcal{M})$ ) along $\sigma, D_{\xi}^{T}(u)$ (resp. $D_{\xi}^{\nu}(u)$ ) is given by $P_{x}\left(u^{\prime}(0)\right)$ (resp. $P_{x}^{\perp}\left(u^{\prime}(0)\right.$, where $P_{x}^{\perp}$ is the orthogonal projection of $\mathbb{R}^{N}$ on $\left.\nu(\mathcal{M})_{x}\right)$. Since the Gauss map $P$ is a smooth map into a vector space, it has a well-defined differential

$$
d P: T \mathcal{M} \rightarrow L\left(\mathbb{R}^{N}, \mathbb{R}^{N}\right)
$$

For $v \in \nu(\mathcal{M})_{x}$ we define $A_{v}: T \mathcal{M}_{x} \rightarrow \mathbb{R}^{N}$ to be the linear map $A_{v}(\xi)=$ $d P_{x}(\xi) v$. Then it is easy to see

4.21. Proposition. Given $\xi$ in $T \mathcal{M}_{x}$ and $v_{0}$ in $\nu(\mathcal{M})_{x}$ let $\sigma:(-\varepsilon, \varepsilon) \rightarrow \mathcal{M}$ be a smooth curve with $\sigma^{\prime}(0)=\xi$ and let $u(t)$ and $v(t)$ be respectively tangent and normal vector fields to $\mathcal{M}$ along $\sigma$, with $v(0)=v_{0}$. Let $P v_{0}$ denote the section $x \rightarrow P_{x}\left(v_{0}\right)$ of $T \mathcal{M}$. Then:

(i) $A_{v_{0}}(\xi)=-P_{x} v^{\prime}(0)$; hence each $A_{v}$ maps $T \mathcal{M}_{x}$ to itself,

(ii) $A_{v_{0}}(\xi)=D_{\xi}^{T}\left(P v_{0}\right)$,

(iii) $\left\langle A_{v_{0}}(\xi), u(0)\right\rangle=\left\langle v_{0}, u^{\prime}(0)\right\rangle$.

4.22. Proposition. At a critical point $x$ of a smooth function $f: \mathcal{M} \rightarrow \mathbb{R}$

$$
\operatorname{hess}(f)_{x}=D^{T}(\nabla f)
$$


Proof. Let $X$ and $Y$ be smooth tangent vector fields near $x$, so that

$$
\begin{aligned}
\operatorname{Hess}(f)_{x}(X, Y) & =X_{x}(Y f)=X_{x}\langle\nabla f, Y\rangle \\
& =\left.\frac{d}{d t}\right|_{t=0}\left\langle\nabla f_{\sigma(t)}, Y_{\sigma(t)}\right\rangle \\
& =\left\langle D_{x}^{T}(\nabla f), Y_{x}\right\rangle+\left\langle\nabla f_{x}, D_{x}^{T} Y\right\rangle
\end{aligned}
$$

where $\sigma$ is any smooth curve with $\sigma(0)=x$ and $\sigma^{\prime}(0)=X$. Since $\nabla f_{x}=0$ the result follows.

Given $a$ in $\mathbb{R}^{N}$ define $F^{a}: \mathbb{R}^{N} \rightarrow \mathbb{R}$ and $H^{a}: \mathbb{R}^{N} \rightarrow \mathbb{R}$ by $F^{a}(x)=\frac{1}{2}\|x-a\|^{2}$ and $H^{a}(x)=x \cdot a$; and let $f^{a}$ and $h^{a}$ be their respective restrictions to $\mathcal{M}$. We note that $\left(\nabla F^{a}\right)_{x}=x-a$ and $\left(\nabla H^{a}\right)_{x}=a$. Now in general given $G: \mathbb{R}^{N} \rightarrow \mathbb{R}$ and $g=G \mid \mathcal{M}, \nabla g_{x}=P_{x}\left(\nabla F_{x}\right)$ for $x \in \mathcal{M}$, so it follows from a direct computation that we have:

4.23. Theorem. (i) $\left(\nabla h^{a}\right)_{x}=P_{x} a$, hence $x$ is a critical point of $h^{a}$ if and only if $a$ is in $\nu(\mathcal{M})_{x}$. Moreover in this case hess $\left(h^{a}\right)_{x}=A_{a}$.

(ii) $\left(\nabla f^{a}\right)_{x}=P_{x}(x-a)$ hence $x$ is a critical point of $f^{a}$ if and only if $x-a$ is in $\nu(\mathcal{M})_{x}$. Moreover in this case hess $\left(f^{a}\right)_{x}=I+A_{(x-a)}$.

Because the hessian of $h^{v}$ is self-adjoint, we have

4.24. Corollary. For each $v$ in $\nu(\mathcal{M}) A_{v}$ is a self-adjoint operator on $T \mathcal{M}_{x}$.

In fact $A_{v}$ is the shape operator of $\mathcal{M}$ in the normal direction $v$, and is related to the second fundamental form of $\mathcal{M}$ in $\mathbb{R}^{N}$ by

$$
\mathrm{II}_{v}(X, Y)=\left\langle A_{v} X, Y\right\rangle .
$$

The eigenvalues of $A_{v}$ are the principal curvatures of $M$ at $x$ in the direction $v$.

4.25. Proposition. Given e in $\nu(\mathcal{M})_{x}$ let $v(t)=x+t e$. Then, for all real $t, x$ is a critical point of $f^{v(t)}$ with corresponding hessian $I-t A_{e}$. Thus the nullity of $f^{v(t)}$ at $x$ is just the multiplicity of $t^{-1}$ as a principal curvature of $\mathcal{M}$ at $x$ in the direction $e$. In particular $f^{v(t)}$ is degenerate at $x$ if and only if $t^{-1}$ is a principal curvature of $M$ at $x$ in the direction e. If 1 is not such a principal curvature then $x$ is a nondegenerate critical point of $f^{x+e}$ and its index is

$$
\sum_{0<t<1} \text { nullity of } f^{v(t)} \text { at } x .
$$

Proof. Taking $a=x+t e$ in (ii) of 4.23 gives the first statement, and it is then immediate that the nullity of $f^{v(t)}$ at $x$ is just $\mu\left(t^{-1}\right)$ where $\mu(\lambda)$ denotes the multiplicity of $\lambda$ as an eigenvalue of $A_{e}$. On the other hand the multiplicity of $\lambda$ as an eigenvalue of $\operatorname{hess}\left(f^{x+e}\right)_{x}=I-A_{e}$ is clearly $\mu(1-\lambda)$. 
Since $\lambda<0$ if and only if $1-\lambda>1$ if and only if $1-\lambda$ equals $t^{-1}$ for some $t$ in $(0,1)$, the formula for the index of $f^{x+e}$ at $x$ follows. q.e.d.

We will denote by $Y: \nu(\mathcal{M}) \rightarrow \mathbb{R}^{N}$ the exponential map $(x, v) \rightarrow x+v$ of the normal bundle of $\mathcal{M}$. Since $T(\nu(\mathcal{M}))_{(x, e)}$ is canonically isomorphic to $T \mathcal{M}_{x} \oplus \nu(\mathcal{M})_{x}=\mathbb{R}^{N}, D Y_{(x, e)}$ can be regarded naturally as a linear operator on $\mathbb{R}^{N}$.

4.26. Definition. If $a=Y(x, e)$ then $a$ is called nonfocal for $\mathcal{M}$ with respect to $x$ if $D Y_{(x, e)}$ is a linear isomorphism of $\mathbb{R}^{N}$. If $m=\operatorname{dim}\left(\operatorname{ker}(D Y)_{(x, e)}\right)$ $>0$ then $a$ is called a focal point of multiplicity $m$ for $\mathcal{M}$ with respect to $x$.

4.27. Proposition. For $a=Y(x, e), a$ is a focal for $\mathcal{M}$ with respect to $x$ if and only if $x$ is a degenerate critical point of $f^{a}$; in fact the nullity of $x$ as a critical point of $f^{a}$ is just equal to the multiplicity of a as a focal point of $\mathcal{M}$ with respect to $x$.

Proof. Let $(\sigma(t), v(t))$ be a smooth normal field to $\mathcal{M}$ along the smooth curve $\sigma(t)$ with $\sigma(0)=x$ and $v(0)=e$. Then

$$
\begin{aligned}
D Y_{(x, e)}\left(\sigma^{\prime}(0), v^{\prime}(0)\right) & =\left.\frac{d}{d t}\right|_{t=0}(\sigma(t)+v(t))=\sigma^{\prime}(0)+v^{\prime}(0) \\
& =\sigma^{\prime}(0)+P_{x} v^{\prime}(0)+P_{x}^{\perp} v^{\prime}(0) \\
& =\left(I-A_{e}\right) \sigma^{\prime}(0)+P_{x}^{\perp} v^{\prime}(0)
\end{aligned}
$$

by (i) of 4.21. Taking $\sigma(t) \equiv x$ and $v(t)=e+t v$ gives that $D Y_{(x, e)}$ on $\nu(\mathcal{M})_{x}$ is the identity map, and hence $\operatorname{ker}\left(D Y_{(x, e)}\right)=\operatorname{ker}\left(1-A_{e}\right)$, which by 4.25 is the null space of hess $\left(f^{a}\right)_{x}$.

4.28. Morse Index Theorem. If $\mathcal{M}$ is a compact smooth submanifold of $\mathbb{R}^{N}, x \in \mathcal{M}, e \in \nu(\mathcal{M})_{x}$, and $a=x+e$ is nonfocal with respect to $x$, then $x$ is a nondegenerate critical point of the "distance function" $f^{a}: \mathcal{M} \rightarrow \mathbb{R}, v \mapsto$ $\frac{1}{2}\|v-a\|^{2}$, and in fact the index of $x$ as a critical point $f^{a}$ is just equal to the number of focal points of $M$ with respect to $x$ on the segment joining $x$ to a, each counted with its multiplicity.

Proof. Immediate from 4.25 and 4.27 .

\section{The homology groups of isoparametric submanifolds}

In this section we will apply the Morse theory developed in $\S 4$ to calculate the homology groups of isoparametric submanifolds. In particular we will construct Bott-Samelson cycles (generalized Schubert cycles) for the distance functions of isoparametric submanifolds. 
5.1. Theorem. Let $M^{n} \subset \mathbb{R}^{n+k}$ be isoparametric, $a \in \mathbb{R}^{n+k}$, and $f: M \rightarrow$ $\mathbb{R}$ the smooth function defined by $f(x)=\frac{1}{2}\|x-a\|^{2}$. Let $C(f)$ denote the set of critical points of $f$. Then

(1) $x \in C(f)$ if and only if $(a-x) \in \nu(M)_{x}$,

(2) if $x \in C(f)$ then $W \cdot x \subset C(f)$,

(3) if $x \in C(f)$ then the hessian of $f$ at $x$ is $I-\sum_{i}\left\langle(a-x), v_{i}\right\rangle \operatorname{id}_{E_{i}}$,

(4) if a is $W$-regular (or equivalently a nonfocal point of $M$ ) then $f$ is a Morse function and $C(f)$ is a principle orbit of $W$. Moreover for $x \in C(f)$ the index of $f$ at $x$ is the sum of the $m_{j}$ 's such that the line segment $[x, a]$ joining $x$ to a meets $l_{j}(x)$,

(5) if $x_{0} \in C(f)$ and $a \in \sigma$, where $\sigma$ is a simplex of the boundary of a Weyl chamber $\Delta$ of $\nu(M)_{x_{0}}$, then $f$ is a Morse function in the sense of Bott [4]; more specifically the critical submanifold through $x_{0}$ is the isoparametric submanifold $N_{x_{0}, \sigma}$, the nullity of $f$ at $x_{0}$ is $m_{\sigma}$ and the index of $f$ at $x_{0}$ is the sum of the $m_{j}$ 's such that the line segment $\left[x_{0}, a\right]$ meets $l_{j}\left(x_{0}\right)$ at a point other than $a$; and $C(f)=\left\{N_{x, \sigma} \mid x \in W \cdot x_{0}\right\}$,

(6) $f$ has a unique local maximum (minimum) value, and the maximum (minimum) level set of $f$ is connected.

Proof. (1), (2), (3) and the second half of (4) follow from the standard Morse theory (see §4) and (1.9) easily.

Now suppose $x, y \in C(f)$ and $a$ is $W$-regular. Then there are parallel normal fields $t$ and $v$ on $M$ such that $a=x+t(x)=y+v(y)$. By (1.10) the parallel manifold $M_{t}$ is equal to $M_{v}$, it is isoparametric, and

$$
\nu(M)_{x}=\nu\left(M_{t}\right)_{a}=\nu\left(M_{v}\right)_{a}=\nu(M)_{y} .
$$

Hence $y \in M \cap \nu(M)_{x}$. However $M \cap \nu(M)_{x}=W \cdot x$, so $y \in W \cdot x$, which proves the first part of (4).

If $v$ is a parallel normal field on $M$ and $a=x_{0}+v\left(x_{0}\right) \in \sigma$ is $W$-singular, then it follows from (2.1) that we have $N_{x, \tau} \subset C(f)$, if $x$ is in $W \cdot x_{0}$ and $\tau$ is open in $V(\sigma)$. Conversely if $y \in C(f)$ then there is a parallel normal field $v^{\prime}$ on $M$ such that $y+v^{\prime}(y)=a$. So $M_{v^{\prime}}=M_{a}$, and there is $x_{1}$ in $W \cdot x_{0}$ such that $a=x_{1}+v^{\prime}\left(x_{1}\right)$. Hence $Y$ belongs to $N_{x_{1}, \sigma}$, which proves (5). Then (6) is a consequence of (4) and (5).

5.2. Theorem. Let $M^{n}$ be an isoparametric submanifold of $\mathbb{R}^{n+k}$ and $x_{0}$ a critical point of $f$ as in (5.1), where $a=x_{0}+v\left(x_{0}\right)$ is $W$-regular for some parallel normal field $v$ on $M$. Suppose

$$
\left\langle v, v_{r}\right\rangle \geq\left\langle v, v_{r-1}\right\rangle \geq \cdots \geq\left\langle v, v_{1}\right\rangle>1>\left\langle v, v_{r+1}\right\rangle \geq \cdots \geq\left\langle v, v_{p}\right\rangle .
$$


Then

(1) $\bigoplus\left\{E_{j}\left(x_{0}\right) \mid i \leq r\right\}$ is the negative space of $f$ at $x_{0}$,

(2) $\left(N_{r}, u_{r}\right)$ is an R-orientiable Bott-Samelson cycle at $x_{0}$ for $f$, where $N_{r}=\left\{\left(y_{1}, \cdots, y_{r}\right) \mid y_{1} \in S_{1}\left(x_{0}\right), y_{2} \in S_{2}\left(y_{1}\right), \cdots, y_{r} \in S_{r}\left(y_{r-1}\right)\right\}, u_{r}: N_{r} \rightarrow$ $M$ is defined by $u_{r}\left(y_{1}, \cdots, y_{r}\right)=y_{r}$, and $S_{i}(x)$ is the leaf of $E_{i}$ through $x$, and $R=\mathbb{Z}$ if all $m_{i}>1$, and $R=\mathbb{Z}_{2}$ otherwise.

Proof. (1) follows from 5.1(3). For (2) it suffices to prove the following three statements:

(a) $y_{0}=\left(x_{0}, \cdots, x_{0}\right)$ is the unique maximum point of $f \circ u_{r}$.

(b) $d\left(u_{r}\right)_{y_{0}}$ maps $T\left(N_{r}\right)_{y_{0}}$ isomorphically onto the negative space of $f$ at $x_{0}$.

(c) If all $m_{i}>1$, then $\left(N_{r}, u_{r}\right)$ is $\mathbb{Z}$-orientable.

To see (b) we note that $N=N_{r}$ is contained in the product of $r$ copies of $M, T N_{y_{0}}=\bigoplus\left\{F_{i} \mid i \leq r\right\}$, where $F_{i}=\left(0, \cdots, E_{j}\left(x_{0}\right), \cdots, 0\right)$ is contained in $\bigoplus\left\{T M_{x_{0}} \mid i \leq r\right\}$, and $d\left(u_{r}\right)_{y_{0}}$ maps $F_{i}$ isomorphically onto $E_{i}\left(x_{0}\right)$.

The homotopy exact sequence for the fibrations implies that if the fiber and the base of a fibration are simply connected then the total space is also simply connected. Hence by induction the iterated sphere bundle $N_{r}$ is simply connected, which proves (c).

Statement (a) follows from the lemma below.

5.3. Lemma. We use the same notation as in 5.2. Then for any $q=$ $\left(y_{1}, \cdots, y_{r}\right)$ in $N_{r}$ there is a continuous piecewise smooth geodesic $\alpha_{q}$ in $\mathbb{R}^{n+k}$ joining a to $y_{r}$ such that the length of $\alpha_{q}$ is $\left\|x_{0}-a\right\|$, and $\alpha_{q}$ is smooth if and only if $q=\left(x_{0}, \cdots, x_{0}\right)$.

Proof. Let $[x y]$ denote the line segment joining $x$ and $y$ in $\mathbf{R}^{n+k}$. Then

$$
\left[a x_{0}\right]=\left[a z_{1}\right] \cup\left[z_{1} z_{2}\right] \cup \cdots \cup\left[z_{r} x_{0}\right]
$$

where $z_{i} \in l_{i}\left(x_{0}\right) \cap\left[x_{0} a\right]$. Let $a_{i}=\left(y_{i}+v\left(y_{i}\right)\right)$, and $z_{j}(i) \in l_{j}\left(y_{i}\right) \cap\left[y_{i} a_{i}\right]$, so $z_{1}(1)=z_{1}$. Since $y_{j} \in S_{j}\left(y_{j-1}\right), z_{j}(j-1)=z_{j}(j)$. Then

$$
\alpha_{q}=\left[a z_{1}\right] \cup\left[z_{1}(1), z_{2}(1)\right] \cup\left[z_{2}(2), z_{3}(2)\right] \cup \cdots \cup\left[z_{r}(r), y_{r}\right]
$$

satisfies the properties of the lemma.

5.4. Theorem. Let $M^{n}$ be an isoparametric submanifold of $\mathbb{R}^{n+k}$ with $W$ and $\left\{m_{i}\right\}$ the associated Weyl group and multiplicities. Choose any point a in $\mathbf{R}^{n+k}$ that is nonfocal for $M$ and define $f: M \rightarrow \mathbb{R}$ by $f(x)=\frac{1}{2}\|x-a\|^{2}$.

(1) If all the multiplicities $m_{i}$ are greater than 1 , then $M$ is simply connected, $H_{*}(M, \mathbb{Z})$ has no torsion, the rank of $H_{i}(M, \mathbb{Z})$ is the number of critical points of $f$ of index $i$ (which can be computed directly from the marked Dynkin diagram of $M$ as stated in Theorem 5.1(4)), and the rank of $H_{*}(M, \mathbb{Z})$ is $|W|$, the order $W$. 
(2) In any case $\operatorname{dim} H_{i}\left(M, \mathbb{Z}_{2}\right)$ is the number of the critical points of $f$ of index $i$ and $\operatorname{dim} H_{*}\left(M, \mathbb{Z}_{2}\right)=|W|$.

A compact submanifold of the Euclidean space is tight (resp. taut) if every nondegenerate height (distance) function is tight. We refer to [17], [15], [12] and [32] for detailed study of tight and taut embeddings. As a consequence of 5.4 we have

5.5. Corollary. A compact isoparametric submanifold is spherically tight, so it is taut.

5.6. Remark. For $M^{n} \subset S^{n+1} \subset \mathbb{R}^{n+2}$, Theorem 5.4 was proved by Münzner in [35], and Corollary 5.5 was proved by Cecil and Ryan in [14].

Using 2.1(vi) and an argument similar to that in 5.4, we obtain:

5.7. Theorem. Suppose $M^{n} \subset \mathbb{R}^{n+k}$ is isoparametric, $v$ is a parallel normal field on $M$, and $x_{0}^{*}=x_{0}+v\left(x_{0}\right)$ is in $\sigma$, where $\sigma$ is a simplex of the boundary of the Weyl chamber $\Delta_{x_{0}}$. Let $M_{x_{0}^{*}}$ be the (focal) parallel manifold to $M$, through $x_{0}^{*}$, a a $W$-regular point, and $x_{0}^{*}$ a critical point of $f: M_{x_{0}^{*}} \rightarrow \mathbb{R}$ where $f(x)=\frac{1}{2}\|x-a\|^{2}$. Then

(i) $\left(x_{0}^{*}-a\right) \in \nu\left(M_{x_{0}^{*}}\right)_{x_{0}^{*}}$,

(ii) the set of critical points of $f$ is the $W$-orbit of $x_{0}^{*}$,

(iii) if $q$ is a critical point of $f$ on $M_{x_{0}^{*}}$, then $\operatorname{ind}(f)_{q}=\sum\left\{m_{j} \mid j\right.$ is not in $I(a, \sigma)$ and $[a q]$ meets $\left.l_{j}(a)\right\}$,

(iv) if all $m_{j}>1$ for $j$ not in $I(a, \sigma)$, then $M_{x_{0}^{*}}$ is simply connected, $H_{*}\left(M_{x_{0}^{*}}, \mathbb{Z}\right)$ has no torsion and its rank is $|W| /\left|W_{\sigma}\right|$,

(v) in any case the dimension of $H_{*}\left(M_{x_{0}^{*}}, \mathbb{Z}_{2}\right)$ is $|W| /\left|W_{\sigma}\right|$.

5.8. Corollary. Every parallel manifold of an isoparametric submanifold (including focal manifolds) is taut.

5.9. Remark. As noted in 1.13 , homogeneous isoparametric submanifolds and their focal manifolds are just the orbits of the isotropy representations of symmetric spaces. These orbits are precisely the so-called $R$-spaces of Kobayashi and Takeuchi [31]. For these homogeneous spaces Theorems 5.4 and 5.7 were proved by Bott and Samelson [5], and their corollaries were proved by Kobayashi and Takeuchi [31].

\section{The cohomology ring of isoparametric submanifolds}

In this section we determine the ring structure of $H^{*}(M, R)$ for an irreducible isoparametric submanifold $M$ with $k \geq 2$. We will adopt the following convention for $R$ : if all $m_{i}$ are even (i.e. $m_{1}=m_{2}=\mu \in\{2,4,8\}$ ) then $R=\mathbb{Z}$ while if some $m_{i}$ is odd then $R=\mathbb{Z}_{2}$. Note that this implies $H^{*}(M, R)$ is commutative. 
Suppose $M^{n} \subset \mathbb{R}^{n+k}$ is isoparametric, $E_{1}, \cdots, E_{p}$ are its curvature distributions, $x_{0} \in M$, and $a$ is a $W$-regular point in the Weyl chamber $\Delta_{x_{0}}$. Let $\bar{U}$ be the Weyl chamber of the associated Weyl group $W$ on $\nu(M)_{x_{0}}$ containing $-x_{0}$, and $x_{1} \in \bar{U} \cap M$. Then $x_{1}$ is the maximum point of the distance function $f(x)=\frac{1}{2}\|x-a\|^{2}$. If $(N, u)$ is a Bott-Samelson cycle for $f$ at $x_{1}$ (e.g. that constructed in Theorem 5.2), then $u: N \rightarrow M$ is a map of degree 1. Therefore:

6.1. Proposition. The map $u^{*}: H^{*}(M, R) \rightarrow H^{*}(N, R)$ is an injective ring homomorphism and the image of $u^{*}$ is an additive direct summand of $H^{*}(N, R)$.

We note that $N$ is an iterated sphere bundle, so its cohomology can be obtained from the Gysin sequence. First we will study the cohomology of the following iterated sphere bundles: let $S_{j}(x)$ denote the leaf (sphere) of the curvature distribution $E_{i}$ through $x$, and for $r \leq p$ define

$$
N_{r}=\left\{\left(y_{1}, \cdots, y_{r}\right) \mid y_{1} \in S_{1}\left(x_{0}\right), y_{2} \in S_{2}\left(y_{1}\right), \cdots, y_{r} \in S_{r}\left(y_{r-1}\right)\right\},
$$

$\pi_{r-1}: N_{r} \rightarrow N_{r-1}$ is the natural projection map

$$
\left(y_{1}, \cdots, y_{r}\right) \rightarrow\left(y_{1}, \cdots, y_{r-1}\right)
$$

$\pi_{p, r}: N_{p} \rightarrow N_{r}$ is the projection

$$
\left(y_{1}, \cdots, y_{p}\right) \rightarrow\left(y_{1}, \cdots, y_{r}\right) .
$$

Then $N_{1}=S_{1}\left(x_{0}\right)$ and $\pi_{r-1}$ is a fibration with the $S_{r}\left(y_{r-1}\right)$ as fibers, so $N_{r}$ is an iterated sphere bundle. By the Gysin sequence we have

$$
H^{*}\left(N_{r}, R\right)=\pi_{r-1}^{*}\left(H^{*}\left(N_{r-1}, R\right)\right) \oplus \xi_{r} \cdot \pi_{r-1}^{*}\left(H^{*}\left(N_{r-1}, R\right)\right),
$$

where $\xi_{r} \mid S_{r}\left(y_{r-1}\right)$ is a generator of $H^{m_{r}}\left(S_{r}\left(y_{r-1}\right), R\right)$. To simplify notation we will just write $\xi_{r}$ for the pull back $\pi_{p, r}^{*}\left(\xi_{r}\right)$. Then it follows by induction that $H^{*}\left(N_{r}, R\right)$ is a free module, generated by $\xi_{1}, \cdots, \xi_{r}$ and $\left\{\xi_{i_{1}} \cdot \xi_{i_{2}} \cdots \xi_{i_{j}} \mid 1 \leq i_{1}<\cdots<i_{j} \leq r\right.$ and $\left.j \leq r\right\}$ forms an $R$-module base for $H^{*}\left(N_{r}, R\right)$. To determine the relation among the $\xi_{i}$ 's we need only express $\xi_{i}^{2}$ in terms of the above basis. We first recall the diffeomorphism $\varphi_{i}$ of $M$ defined by: $\varphi_{i}(x)$ is the antipodal point of $x$ in the sphere $S_{i}(x)$. Then $\varphi_{i}$ induces a diffeomorphism $\bar{\varphi}_{i}$ on $N_{i}$ by

$$
\bar{\varphi}_{i}\left(\left(y_{1}, \cdots, y_{i}\right)\right)=\left(y_{1}, \cdots, \varphi_{i}\left(y_{i}\right)\right), \quad \text { and } \quad \pi_{i-1} \circ \bar{\varphi}_{i}=\pi_{i-1} \text {. }
$$

Let $a_{i}, i \leq r$, be the homology class determined by the cycle

$$
\left\{\left(y_{1}, \cdots, y_{r}\right) \mid y_{1}=\cdots=y_{i-1}=x_{0}, \text { and } y_{i}=\cdots=y_{r} \in S_{i}\left(x_{0}\right)\right\} \text {. }
$$

Then $a_{1}, \cdots, a_{r}$ forms a basis for $H_{m_{1}}\left(N_{r}, R\right) \oplus H_{m_{2}}\left(N_{r}, R\right)$. Then the $\xi_{i}$ 's can be chosen as the dual basis for $H^{m_{1}}\left(N_{r}, R\right) \oplus H^{m_{2}}\left(N_{r}, R\right)$ so that $\xi_{i}\left(a_{j}\right)=\delta_{i j}$. 
6.2. Lemma. For $j<r$ there exist $\beta_{r j} \in R$ such that

(i) $\left(\bar{\varphi}_{r}\right)_{*}\left(a_{j}\right)=a_{j}+\beta_{r j} a_{r}$ for $j<r$,

(ii) $\left(\bar{\varphi}_{r}\right)=-\xi_{r}+\sum_{j<r} \beta_{r j} \xi_{j}$.

Proof. For $j<r$, since $a_{1}, \cdots, a_{r}$ forms a base for

$$
H^{m_{1}}\left(N_{r}, R\right) \oplus H^{m_{2}}\left(N_{r}, R\right),\left(\bar{\varphi}_{r}\right)_{*}\left(a_{j}\right)
$$

is a linear combination of $a_{1}, \cdots, a_{r}$ in $N_{r}$ with coefficients in $R$. Because $\pi_{r-1} \circ \bar{\varphi}_{r}=\pi_{r-1}$, we have

$$
\left(\pi_{r-1}\right)_{*}\left(\bar{\varphi}_{r}\right)_{*}\left(a_{j}\right)=\left(\pi_{r-1}\right)_{*}\left(a_{j}\right) \text { in } N_{r-1} .
$$

So

$$
\left(\bar{\varphi}_{r}\right)_{*}\left(a_{j}\right)=a_{j}+\beta_{r j} a_{r} \quad \text { for some } \beta_{r j} \in R \text {. }
$$

Since $\left(\bar{\varphi}_{r}\right)^{*}\left(\xi_{r}\right)\left(a_{j}\right)=\xi_{r}\left(\left(\varphi_{r}\right)_{*}\left(a_{j}\right)\right)=\beta_{r j}$, and $\left(\bar{\varphi}_{r}\right)^{*}\left(\xi_{r}\right)\left(a_{r}\right)=\xi_{r}\left(\left(\varphi_{r}\right)_{*}\left(a_{r}\right)\right)$ $=-1$ we have (ii).

6.3. Lemma. $H^{*}\left(N_{s}, R\right)=R\left[\xi_{1}, \cdots, \xi_{s}\right] / I$, where $I$ is the ideal generated by

$$
\eta_{r}=\xi_{r}^{2}-\sum_{j<r} \beta_{r j} \xi_{r} \xi_{j}, \quad r \leq s .
$$

Moreover if $m_{r} \neq m_{j}$ then $\beta_{r j}=0$, and if $m_{r}=m_{j}$ then $\beta_{r j}$ is the intersection number of $\left(\bar{\varphi}_{r}\right)_{*}\left(a_{j}\right)$ and the Poincaré dual of $\xi_{r}$ in $N_{r}$.

Proof. Because $\pi_{r-1} \circ \bar{\varphi}_{r}=\pi_{r-1}$ we have $\tau \in\left(\pi_{r-1}\right)^{*}\left(H^{*}\left(N_{r}, R\right)\right)$ if and only if $\tau$ is invariant under $\left(\bar{\varphi}_{r}\right)^{*}$. Since $\left(\bar{\varphi}_{r}\right)^{2}=\mathrm{id}$ and $(-1)^{m_{r}}=1 \mathrm{in} R$, we have

$$
\left(\bar{\varphi}_{r}\right)^{*}\left(\xi_{r} \cdot \bar{\varphi}_{r}^{*}\left(\xi_{r}\right)\right)=\bar{\varphi}_{r}^{*}\left(\xi_{r}\right) \cdot \xi_{r}=\xi_{r} \cdot \bar{\varphi}_{r}^{*}\left(\xi_{r}\right) .
$$

Let $v=\xi_{r} \cdot \bar{\varphi}_{r}^{*}\left(\xi_{r}\right)$. Then there is $u$ in $H^{*}\left(N_{r-1}, R\right)$ such that $v=\pi_{r-1}^{*}(u)$. We claim that $v=0$. To see this we note that $s_{r-1}\left(y_{1}, \cdots, y_{r-1}\right)=$ $\left(y_{1}, \cdots, y_{r-1}, y_{r-1}\right)$ is a cross section of the sphere bundle $\pi_{r-1}: N_{r} \rightarrow N_{r-1}$, i.e., $\pi_{r-1} \circ s_{r-1}=\mathrm{id}$, and $s_{r-1}^{*}\left(\xi_{r}\right)=0$. Hence

$$
\begin{aligned}
s_{r-1}^{*}(v) & =s_{r-1}^{*}\left(\xi_{r} \cdot \bar{\varphi}_{r}^{*}\left(\xi_{r}\right)\right)=0 \\
& =s_{r-1}^{*}\left(\pi_{r-1}^{*}(u)\right)=\operatorname{id}^{*}(u)=u .
\end{aligned}
$$

This proves that $v=0$, i.e., $\xi_{r}^{2}-\sum_{j<r} \beta_{r j} \xi_{r} \xi_{j}=0$. q.e.d.

In the following we will determine $\beta_{i j}$ in terms of the geometry of $M$.

6.4. Lemma. Let $b_{i}$ denote the homology class of the sphere $S_{i}\left(x_{0}\right)$. Then

(i) $\left(\varphi_{i}\right)_{*}\left(b_{j}\right)=\operatorname{sgn}\left(1-2\left\langle v_{i}, v_{j}\right\rangle /\left\|v_{i}\right\|^{2}\right) b_{\rho^{i}(j)}$, where $\rho: W \rightarrow S_{p}$ is the representation as in 1.5 ,

(ii) there are $\beta_{i j}$ in $R$ such that $\left(\varphi_{i}\right)_{*}\left(b_{j}\right)=b_{j}+\beta_{i j} b_{i}$, and in particular if $m_{i} \neq m_{j}$ then $\beta_{i j}=0$. 
Proof. Let $X$ denote the position map of $M^{n}$ in $\mathbb{R}^{n+k}$. Then $\varphi_{i}=X+$ $2 v_{i} /\left\|v_{i}\right\|^{2}$ and $d \varphi_{i}\left(E_{j}\right)=\operatorname{sgn}\left(1-2\left\langle v_{i}, v_{j}\right\rangle /\left\|v_{i}\right\|^{2}\right)\left(E_{\rho^{i}(j)}\right)$, which proves (i). Next we define

$$
\begin{gathered}
N_{2}=\left\{\left(y_{i}, y_{j}\right) \mid y_{i} \in S_{i}\left(x_{0}\right), y_{j} \in S_{j}\left(y_{i}\right)\right\} \\
\pi: N_{2} \rightarrow M, \quad \text { where } \pi\left(\left(y_{i}, y_{j}\right)\right)=y_{j}, \\
a_{i}=\left\{\left(y_{i}, y_{i}\right) \mid y_{i} \in S_{i}\left(x_{0}\right)\right\}, \quad a_{j}=\left\{\left(x_{0}, y_{j}\right) \mid y_{j} \in S_{j}\left(x_{0}\right)\right\} .
\end{gathered}
$$

then $\pi\left(a_{i}\right)=b_{i}$ and $\pi\left(a_{j}\right)=b_{j}$ and the lemma follows from 6.2(i). q.e.d.

Therefore we have the following generalization of a theorem of Bott and Samelson:

6.5. Theorem. Let $M^{n}$ be isoparametric in $\mathbb{R}^{n+k}, W$ its Weyl group, $b_{i}$ the homology class of the leaf $S_{i}(x)$ through $x$ of the curvature distribution $E_{i}$, and $(N, u)$ the Bott-Samelson cycle at the maximum point $x_{0}$ for $f(x)=$ $\frac{1}{2}\|x-a\|^{2}$. Suppose we arrange the indices so that

$$
N=\left\{\left(y_{1}, \cdots, y_{p}\right) \mid y_{1} \in S_{1}\left(x_{0}\right), y_{2} \in S_{2}\left(y_{1}\right), \cdots, y_{p} \in S_{p}\left(y_{p-1}\right)\right\}
$$

and $u: N \rightarrow M$ is defined by $u\left(\left(y_{1}, \cdots, y_{p}\right)\right)=y_{p}$. Then

(i) there are $\beta_{i j}$ in $R$ such that $\left(\varphi_{i}\right)_{*}\left(b_{j}\right)=b_{j}+\beta_{i j} b_{i}$,

(ii) $H^{*}(M, R)$ is a subring and an additive direct summand of the ring $A=R\left[\xi_{1}, \cdots, \xi_{p}\right] / I$, where $I$ is the ideal generated by

$$
\eta_{r}=\xi_{r}^{2}-\sum_{r>j} \beta_{r j} \xi_{r} \xi_{j}, \quad r \leq p .
$$

In the following we will assume that $M$ is irreducible with $k>1$ and all the $m_{i}$ 's are even, i.e., $m_{1}=m_{2}=\mu \in\{2,4,8\}$. To determine the ring structure of $H^{*}(M, \mathbb{Q})$ more explicitly we need the following well-known fact:

6.6. Theorem. Suppose $G$ is a finite group acting freely on $M$. Then

(i) $H^{*}(M / G, \mathbb{Q})=H^{*}(M, \mathbb{Q})^{G}$,

(ii) $\chi(M / G)=\chi(M) /|G|$, where $\chi$ denote the Euler characteristic.

Then it follows from Theorem 5.4(1) that $\chi(M)=|W|$ and we have

6.7. Corollary. Suppose $M^{n} \subset \mathbb{R}^{n+k}$ is an irreducible isoparametric submanifold with even multiplicities, and $W$ is the associated Weyl group. Then $H^{i}(M, \mathbb{Q})^{W}=0$ for all $i>0$.

The following is a known result for Weyl groups that follows easily from Borel's theorem on the cohomology of $G / T$ :

6.8. Proposition. Suppose $W$ is a rank $k$ Weyl group acting on $\mathbb{R}^{k}$. Then

(i) the dimension of $\mathbb{Q}\left[x_{1}, \cdots, x_{k}\right] /\left\langle\mathbb{Q}^{+}\left[x_{1}, \cdots, x_{k}\right]\right\rangle^{W}$ is $|W|$, the order of the group $W$, where $\left\langle\mathbb{Q}^{+}\left[x_{1}, \cdots, x_{k}\right]\right\rangle^{W}$ is the ideal generated by the nonconstant $W$-invariant homogeneous polynomials of $\mathbb{R}^{k}$, 
(ii) $\operatorname{dim}\left(\mathbb{Q}\left[x_{1}, \cdots, x_{k}\right]^{W_{a}} /\left\langle\mathbb{Q}^{+}\left[x_{1}, \cdots, x_{k}\right]\right\rangle^{W}\right)=|W| /\left|W_{a}\right|$, where $W_{a}$ is the isotropy subgroup of a in $\mathbf{R}^{k}$.

Suppose $x_{0}$ is the maximum point of $f^{a}, \Delta_{x_{0}}$ and $I\left(x_{0}, \Delta_{x_{0}}\right)=$ $\{i(1), \cdots, i(k)\}$ as in 1.7. Then the homology classes $b_{i(j)}$ (representing $\left.S_{i(j)}\left(x_{0}\right)\right)$ is the Bott-Samelson cycle at the critical point $\varphi_{i(j)}\left(x_{0}\right)$ of $f^{x_{0}}$. Hence $b_{i(1)}, \cdots, b_{i(k)}$ form a set of generators of $H_{\mu}(M, \mathbb{Z})$. Therefore $W$ induces a group homomorphism $\sigma: W \rightarrow \mathrm{GL}(k, \mathbb{Z})$, where $\sigma\left(\varphi_{i}\right)=B_{i}$, the matrix of $\varphi_{i^{*}}$ on $H_{\mu}(M, \mathbb{Z})$ relative to the basis $b_{i(1)}, \cdots, b_{i(k)}$.

6.9. Lemma. $\sigma: W \rightarrow \mathrm{GL}(k, \mathbb{Z})$ is injective.

Proof. Since $W$ and $\sigma(W)$ are Weyl groups, which are determined by the generators and relations, $\sigma$ is injective provided it is injective when restricted to any rank 2 Weyl subgroup of $W$. But every rank 2 Weyl subgroup corresponds to some $(k-2)$-simplex of a Weyl chamber of $W$. Hence by the Slice Theorem 2.1, it suffices to prove this lemma for $k=2$, i.e., $W=D_{g}$ (the dihedral group of order $2 g$ ), where $g=2,3,4$, or 6 . Recall that the ordering of the reflection hyperplanes $l_{i}$ has been set in 6.5. So $I\left(x_{0}, \Delta_{x_{0}}\right)=\{1, g\}$. It follows from $6.4(1)$ that we have $\left(\varphi_{1}\right)_{*}\left(b_{g}\right)=b_{2}$, and $\left(\varphi_{1}\right)_{*}\left(b_{1}\right)=-b_{2}$. So $b_{1}, b_{2}$ form a basis for $H_{\mu}(M, \mathbf{Z})$. Using 6.4 again, we have

$$
\begin{gathered}
\sigma\left(\varphi_{1}\right)=B_{1}=\left[\begin{array}{cc}
-1 & a \\
0 & 1
\end{array}\right], \quad \sigma\left(\varphi_{2}\right)=B_{2}=\left[\begin{array}{cc}
1 & 0 \\
b & -1
\end{array}\right], \\
\sigma\left(\varphi_{1} \varphi_{2}\right)=B=\left[\begin{array}{cc}
a b-1 & -a \\
b & -1
\end{array}\right],
\end{gathered}
$$

where $a=\beta_{12}$ and $b=\beta_{21}$. Note that $B \neq \mathrm{id}$, so if $\sigma$ is not injective then we have $g=4$ or $6,1<o(B)<g$ and $o(B)$ is a divisor of $g$, where $o(B)$ denotes the order of $B$. Therefore we have either $o(B)=2$, or $o(B)=3$ and $g=6$. In each case we will derive a contradiction as follows:

(i) $o(B)=2$. Since $\operatorname{det}(B)=1$ and $o(B)=2$, we have $\operatorname{tr}(B)=-2=a b-2$. Hence $a b=0$, and

$$
B=\left[\begin{array}{cc}
-1 & 0 \\
b & -1
\end{array}\right] \text { or } \quad B=\left[\begin{array}{cc}
-1 & -a \\
0 & -1
\end{array}\right]
$$

$B^{2}=1$ implies that $B$ must be -1 , i.e. $a=b=0$. So $\sigma(\varphi)$ is a diagonal matrix for all $\varphi$ in $W$, all $\beta_{r j}=0$, and the ideal $I$ in 6.5 is generated by $\xi_{i}^{2}=0$ for $i \leq g$.

Let $W_{0}$ denote the kernel of $\sigma$. Then by 6.6(ii) we have $\chi\left(M / W_{0}\right)=$ $|W| /\left|W_{0}\right|=4$, and $H^{*}\left(M / W_{0}, \mathbb{Q}\right)=H^{*}(M, \mathbb{Q})^{W_{0}}$ has dimension 4. Let $\omega_{1}, \omega_{2}$ be the basis for $H^{\mu}(M, \mathbb{Q})$ dual to $b_{1}, b_{2}$. Then $\omega_{1}, \omega_{2}$ are $W_{0}$-invariant and hence $\operatorname{dim}\left(H^{\mu}(M, \mathbb{Q})^{W_{0}}\right)=2$ It is obvious that $\omega_{1} \wedge \omega_{2}$ is $W_{0}$-invariant. And it follows from the fact that $I$ is generated by $\xi_{i}^{2}=0$ for $i \leq g$ that $\omega_{1} \wedge \omega_{2}$ 
is nonzero. So $\operatorname{dim}\left(H^{2 \mu}(M, \mathbb{Q})^{W_{0}}\right) \geq 1$. But

$$
\operatorname{dim}\left(H^{0}(M, \mathbb{Q})^{W_{0}}\right)=\operatorname{dim}\left(H^{g \mu}(M, \mathbb{Q})^{W_{0}}\right)=1,
$$

which implies $\chi\left(M / W_{0}\right) \geq 5$ a contradiction.

(ii) $o(B)=3$, and $g=6$. Since $B^{3}=I, a b-2=\operatorname{tr}(B)=2 \cos (2 \pi / 3)=-1$, so $a b=1$. But $a$ and $b$ are integers so we may assume $a=b=1$. Next we use 6.4(i) and (ii) to express $b_{i}, i>2$, as a linear combination of $b_{1}, b_{2}$ with integer coefficients as follows:

$$
\begin{aligned}
& \left(\varphi_{1}\right)_{*}\left(b_{2}\right)=b_{6}=b_{1}+b_{2} \\
& \left(\varphi_{2}\right)_{*}\left(b_{1}\right)=-b_{3}=b_{1}+b_{2}, \\
& \left(\varphi_{1}\right)_{*}\left(b_{3}\right)=b_{5}=\left(\varphi_{1}\right)_{*}\left(-b_{1}-b_{2}\right)=b_{1}-\left(b_{1}+b_{2}\right)=-b_{2}, \\
& \left(\varphi_{2}\right)_{*}\left(b_{6}\right)=b_{4}=\left(\varphi_{2}\right)_{*}\left(b_{1}+b_{2}\right)=b_{1}, \\
& \left(\varphi_{3}\right)_{*}\left(b_{1}\right)=-b_{5}=b_{2}=b_{1}+\beta_{31} b_{3}=b_{1}+\beta_{31}\left(-b_{1}-b_{2}\right) .
\end{aligned}
$$

Since $b_{1}, b_{2}$ are linearly independent, the last equation gives a contradiction.

6.10. Corollary. With the notations as in $6.4, \beta_{i j} \beta_{j i}=4 \cos ^{2} \theta_{i j}$, where $\theta_{i j}$ is the angle between $l_{i}$ and $l_{j}$, i.e., if $v_{1}, \cdots, v_{k}$ is a simple root system for $W$ then $\left(\beta_{i j}\right)$ is a Cartan matrix of $W$.

6.11. Corollary. The ring structure of $H^{*}(M, \mathbb{Q})$ depends only on $W$ and $\mu\left(=m_{1}=m_{2}\right)$. Moreover $H^{\mu}(M, \mathbb{Q})$ generates $H^{*}(M, \mathbb{Q})$.

Proof. Suppose $v_{1}, \cdots, v_{k}$ is a simple root system for $W$, so that $\left(\varphi_{j}\right)_{*}\left(b_{j}\right)$ $=b_{j}+\beta_{i j} b_{i}$, where $\left(\beta_{i j}\right)$ is the Cartan matrix of $W$, and there exist integers $\alpha_{m i}$ which depend only on $W$ and $\left(\beta_{i j}\right)$ such that

$$
b_{m}=\sum_{i \leq k} \alpha_{m i} b_{i}
$$

Let $\omega_{1}, \cdots, \omega_{k}$ be the dual basis of $b_{1}, \cdots, b_{k}$ in $H^{\mu}(M, \mathbb{Z})$, i.e., $\omega_{i}\left(b_{j}\right)=\delta_{i j}$. Then

$$
\omega_{i}=\xi_{i}+\sum_{m>k} \alpha_{m i} \xi_{m}
$$

These discussions imply that the ring $A$ defined in 6.5 and its subring generated by $\omega_{1}, \cdots, \omega_{k}$ depend only on $W$ and $\mu$. Now let $G$ be a compact connected Lie group which has $W$ as its Weyl group and $T$ as a maximal torus. Then $G / T$ is isoparametric in $\mathfrak{g}$. By a theorem of Borel [3] $H^{2}(G / T, \mathbb{Q})$ generates $H^{*}(G / T, \mathbb{Q})$, hence the subring generated by $\omega_{1}, \cdots, \omega_{k}$ has dimension $|W|$, which is the dimension of $H^{*}(M, \mathbb{Q})$. q.e.d.

Therefore we have the following generalization of the Borel theorem to isoparametric submanifolds:

6.12. Theorem. Let $M^{n} \subset \mathbb{R}^{n+k}$ be an irreducible isoparametric submanifold with even multiplicities, $W$ is its associated Weyl group, $E_{1}, \cdots, E_{p}$ 
the curvature distributions, and $v_{1}, \cdots, v_{p}$ the curvature normals of $M$. Suppose $v_{1}, \cdots, v_{k}$ forms a simple root system for $W$, and $\tau_{i}$ denotes the Euler class of the vector bundle $E_{i}$ on $M$. Then

$$
H^{*}(M, \mathbb{Q})=\mathbb{Q}\left[\tau_{1}, \cdots, \tau_{k}\right] /\left\langle\mathbb{Q}^{+}\left[\tau_{1}, \cdots, \tau_{k}\right]^{W}\right\rangle .
$$

Proof. Using 6.8, it suffices to prove that $\tau_{1}, \cdots, \tau_{k}$ form a basis of $H^{\mu}(M, \mathbb{Q})$, i.e., $\operatorname{det}\left(t_{i j}\right) \neq 0$, where $\tau_{i}=\sum_{j \leq k} t_{i j} \omega_{j}$. We have $\varphi_{i}^{*}\left(\tau_{i}\right)=-\tau_{i}$. But we also have

$$
\begin{aligned}
\varphi_{i}^{*}\left(\tau_{i}\right)\left(b_{j}\right) & =\tau_{i}\left(\left(\varphi_{i}\right)_{*}\left(b_{j}\right)\right)=\tau_{i}\left(b_{j}+\beta_{i j} b_{i}\right)=t_{i j}+2 \beta_{i j} \\
& =-\tau_{i}\left(b_{j}\right)=-t_{i j},
\end{aligned}
$$

so $\left(t_{i j}\right)=\left(\beta_{i j}\right)$, which is a rank $k$ Cartan matrix (because of 6.9). Hence $\operatorname{det}\left(t_{i j}\right) \neq 0$. q.e.d.

By a theorem of Chevalley [18] the ring of $W$-invariant polynomials on $\mathbb{R}^{k}$ is a polynomial ring with $k$ homogeneous generators $u_{1}, \cdots, u_{k}$.

6.13. Corollary. The Poincaré polynomial of $M$ is

$$
P(M, \mathbb{R})=\left(1-t^{\mu}\right)^{k} \prod_{i=1}^{k}\left(1-t^{\mu d_{i}}\right)^{-1},
$$

where the $d_{i}$ are the degrees of the generators $u_{1}, \cdots, u_{k}$ of $\mathbb{R}\left[x_{1}, \cdots, x_{k}\right]^{W}$.

Suppose $a=x_{0}+v\left(x_{0}\right) \in \sigma$, where $\sigma$ is a simplex of the boundary of the Weyl chamber $\Delta_{x_{0}}$. Then for any $\varphi \in W_{a}$ we have $\pi_{v} \circ \varphi=\pi_{v}$. Hence $\pi_{v}^{*}\left(H^{*}\left(M_{a}, \mathbb{Q}\right)\right) \subset H^{*}(M, \mathbb{Q})^{W_{a}}$. Then it follows from 5.7(iv) and 6.8 that these two vector spaces have the same dimension, hence they are equal. Therefore we have

6.14. Theorem. $H^{*}\left(M_{a}, \mathbb{Q}\right)=\mathbb{Q}\left[\tau_{1}, \cdots, \tau_{k}\right]^{W_{a}} /\left\langle\mathbb{Q}^{+}\left[\tau_{1}, \cdots, \tau_{k}\right]^{W}\right\rangle$.

\section{References}

[1] U. Abresch, Isoparametric hypersurfaces with four or six distinct principal curvatures, Math. Ann. 264 (1983) 283-302.

[2] C. T. Benson \& L. C. Grove, Finite reflection groups, Bogden S. Quigley, 1971.

[3] A. Borel, Sur la cohomologie des espaces fibrés principaux et desespaces homogénes des groupes de Lie compacts, Ann. of Math. (2) 57 (1953) 115-207.

[4] R. Bott, Non-degenerate critical manifolds, Ann. of Math. (2) 60 (1954) 248-261.

[5] R. Bott \& H. Samelson, Applications of the theory of Morse to symmetric spaces, Amer. J. Math. 80 (1958) 964-1029.

[6] S. Cairns \& M. Morse, Critical point theory in global analysis and differential topology, Academic Press, New York, 1969.

[7] É. Cartan, Familles de surfaces isoparamétrique dans les éspace à courbure constante, Ann. Mat. Pura Appl. 17 (1938) 177-191.

[8] __ Sur des familles remarquables d'hypersurfaces isoparametriques dans les espaces spheriques, Math. Z. 45 (1939) 335-367. 
[9] __ Sur des familles remarquables d'hypersurfaces, C. R. Congres Math. Liege., 1939, 30-41.

[10] _ Sur quelque familles remarquables d'hypersurfaces, des espaces spheriques a 5 et a 9 dimension, Univ. Nac. Tucumán Rev. Ser. A 1 (1940) 50-42.

[11] _ Leçons sur la géométrie des éspaces de Riemann, Gauthier Villars, Paris, 1946.

[12] S. Carter \& A. West, Tight and taut immersions, Proc. London Math. Soc. 25 (1972) 701-720.

[13] _ Isoparametric systems and transnormality, Proc. London Math. Soc. (3) 51 (1985) 520-542.

[14] T. E. Cecil \& P. J. Ryan, Tight spherical embeddings, Lecture Notes in Math., Vol. 838, Springer, Berlin, 1981, 94-104.

[15] _ , Tight and taut immersions of manifolds, Research Notes in Math., Vol. 107, Pitman, Boston, 1985.

[16] S. S. Chern \& C. Chevalley, Élie Cartan and his mathematical work, Bull. Amer. Math. Soc. 58 (1952) 217-250.

[17] S. S. Chern \& R. K. Lashof, On the total curvature of immersed manifolds. I. Amer. J. Math. 79 (1957) 306-318; II, Michigan Math. J. 5 (1958) 5-12.

[18] C. Chevalley, Invariants of finite groups generated by reflections, Amer. J. Math. 77 (1955) 778-782.

[19] L. Conlon, Variational completeness and K-transversal domains, J. Differential Geometry 5 (1971) 135-147.

[20] _ـ A class of variationally complete representations, J. Differential Geometry 7 (1972) 149-160.

[21] J. Dadok, Polar coordinates induced by actions of compact lie groups, Trans. Amer. Math. Soc. 288 (1985) 125-137.

[22] E. B. Dynkin, The structure of semisimple Lie algebras, Uspekhi Mat. Nauk. (N.S.) 2 (1947) 59-127; English transl., Amer. Math. Soc. 17 (1950).

[23] D. Ferus, H. Karcher \& H. F. Münzner, Cliffordalgebren and neue isoparametrische hyperflächen, Math. Z. 177 (1981) 479-502.

[24] D. Ferus \& H. Karcher, Non-rotational minimal spheres and minimizing cones, Comment. Math. Helv. 60 (1985) 247-269.

[25] S. Helgason, Differential geometry and symmetric spaces, Academic Press, New York, 1978.

[26] W. Y. Hsiang, On the compact homogeneous minimal submanifolds Proc. Nat. Acad. Sci. U.S.A. 56 (1966) 5-6.

[27] _ Minimal cones and the spherical Bernstein problem. I, Ann. of Math. (2) 118 (1983); 61-73; II, Invent. Math. 74 (1983) 351-369.

[28] W. Y. Hsiang \& B. H. Lawson, Jr., Minimal submanifolds of low cohomogeneity, J. Differential Geomtry 5 (1971) 1-38.

[29] W. Y. Hsiang, R. S. Palais \& C. L. Terng, The topology and geometry of isoparametric submanifolds in Euclidean spaces, Proc. Nat. Acad. Sci. U.S.A. 82 (1985) 4863-4865.

[30] S. Kobayashi, Imbeddings of homogeneous spaces with minimum total curvature, Tôhoku Math. J. 19 (1967) 63-70.

[31] S. Kobayashi \& M. Takeuchi, Minimal imbeddings of $R$-spaces, J. Differential Geometry 2 (1968) 203-215.

[32] N. H. Kuiper, Minimal total absolute curvature for immersions, Invent. Math. 10 (1970) 209-238.

[33] T. Levi-Civita, Famiglie di superficie isoparametrische nell'ordinario spacio euclideo, Atti Accad. Naz. Lincei. Rend. Cl. Sci. Fis. Mat. Natur. 26 (1937) 355-362.

[34] J. W. Milnor, Morse theory, Annals of Math. Studies, No. 51, Princeton University Press, Princeton, NJ, 1963. 
[35] H. F. Münzner, Isoparametric Hyperflächen in Sphären. I, Math. Ann. 251 (1980) 57-71; II, Math. Ann. 256 (1981) 215-232.

[36] K. Nomizu, Élie Cartan's work on isoparametric families of hypersurfaces, Proc. Sympos. Pure Math., No. 27, Amer. Math. Soc., Providence, RI, 1975, 191-200.

[37] H. Ozeki \& M. Takeuchi, On some types of isoparametric hypersurfaces in spheres. 1, Tôhoku Math. J. 27 (1975) 515-559; 2, Tôhoku Math. J. 28 (1976) 7-55.

[38] R. S. Palais, Morse theory on Hilbert manifold, Topology 2 (1963) 299-340.

[39] __ The principle of symmetric criticality, Comm. Math. Phys. 69 (1979) 19-30.

[40] R. S. Palais \& C. L. Terng, A general canonical form theory, Trans. Amer. Math. Soc. 300 (1987) 771-789.

[41] __ Reduction of variables for minimal submanifolds, Proc. Amer. Math. Soc. 98 (1986) 480-484.

[42] B. Segré, Famiglie di ipersuperficie isoparametrische negli spazi euclidei ad un qualunque numero di demensioni, Atti Accad. Naz. Lincei, Rend. Cl. Sci. Fis. Mat. Natur. 27 (1938) 203-207.

[43] H. Toda, Nodes on the cohomology ring of certain spaces, Proc. Amer. Math. Soc. 14 (1963) 89-95.

[44] C. L. Terng, Isoparametric submanifolds and their Coxeter groups, J. Differential Geometry 21 (1985) 79-107.

UNIVERSITY OF CALIFORNIA, BERKELEY BRANDEIS UNIVERSITY NORTHEASTERN UNIVERSITY 